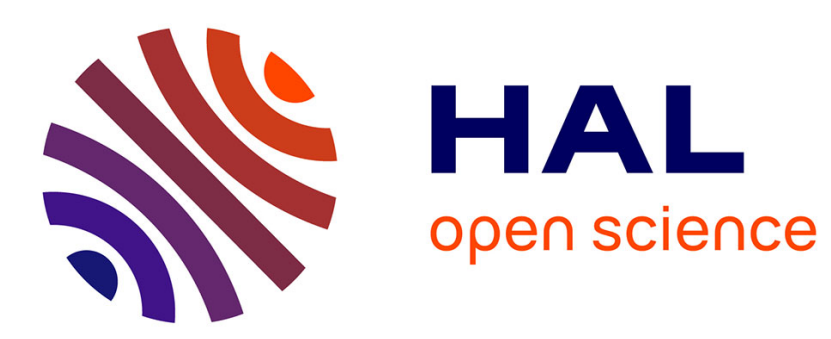

\title{
The concept of strongly interacting groups in self-assembly of soft matter
}

\author{
Alexander Semenov, I. Nyrkova
}

\section{To cite this version:}

Alexander Semenov, I. Nyrkova. The concept of strongly interacting groups in self-assembly of soft matter. European Physical Journal E: Soft matter and biological physics, 2018, 41 (9), pp.103. 10.1140/epje/i2018-11699-2 . hal-03543242

\section{HAL Id: hal-03543242 \\ https://hal.science/hal-03543242}

Submitted on 25 Jan 2022

HAL is a multi-disciplinary open access archive for the deposit and dissemination of scientific research documents, whether they are published or not. The documents may come from teaching and research institutions in France or abroad, or from public or private research centers.
L'archive ouverte pluridisciplinaire HAL, est destinée au dépôt et à la diffusion de documents scientifiques de niveau recherche, publiés ou non, émanant des établissements d'enseignement et de recherche français ou étrangers, des laboratoires publics ou privés. 


\title{
The Concept of Strongly Interacting Groups in Self-Assembly of Soft Matter
}

\author{
I.A.Nyrkova, A.N.Semenov \\ Institut Charles Sadron, CNRS - UPR 22, Université de Strasbourg, \\ 23 rue du Loess, BP 84047, 67034 Strasbourg Cedex 2, France
}

(May 14, 2018)

\begin{abstract}
Amphiphilic molecules in solution typically produce structures coming from cooperative interactions of many synergetically acting functional units. If all essential interactions are weak, the structure can be treated theoretically based on a free energy expansion for small interaction parameters. However, most self-assembling soft matter systems involve strong specific pairwise interactions of functional units leading to qualitatively new structures of highly soluble micellar or fibrillar aggregates. Here we focus on the systems with the so-called strongly interacting groups (SIGs) incorporated into a unimer molecule and discuss the effects of packing frustrations and unimer chirality as well as the origins of spontaneous morphological chirality in the case of achiral unimers. We describe several theoretical approaches (overcoming the limitations of weak interaction models) including the concepts of superstrong segregation, geometrical mismatch and orientational frustration. We also review some recently developed phenomenological theories of surfactant membranes and multiscale hierarchical approaches based on all-atomic modeling of packing structures of amphiphilic molecules with SIGs. In particular, we discuss self-assembling structures in systems possessing simultaneously several distinct types of SIGs: solutions of beta-sheet oligopeptides (showing different fibrillar morphologies), aromatic diamide-ester molecules (forming membranes, helical ribbons and tubules), and triarylamine amide derivatives (producing light-controlled supramolecular nanowires).
\end{abstract}




\section{Introduction}

All known condensed states of matter (including liquid, crystal and amorphous phases, not to mention partially ordered mesophases) are defined by competition between inter-particle attractions and repulsions. A classical theoretical approach in soft matter physics is to take into account two types of interactions: strong and weak. For example, in macromolecular systems covalent bonds and steric repulsions are strong, whereas long-range attractive interactions can be often considered as weak (described with a small interaction $\chi$-parameter). It is also often assumed that the interaction energy between non-neighboring repeat units is typically weak (as compared to $k_{B} T$ ), while the polymer volume fraction is rather low (semidilute polymer regime). These assumptions allow to use the virial expansion and the mean field Edwards approach to derive the smooth monomer concentration profiles and other structural properties of semidilute polymer systems. [1-4]

Similarly, the mean-field Maier-Saupe theory of liquid crystalline systems assumes weak and long-range orientational interactions which can be described with the concept of the molecular 'orientational field'. [5] Further, the classical theories of (poly) electrolyte systems assume weak pairwise Coulombic interactions allowing to use a self-consistent field approach (Debye-Hückel approximation). [6] Most theories of block-copolymer micelles assume that chemical architecture of the amphipathic building blocks is permanent, whereas interactions between distant monomer units are effectively weak. [7] In the theories of polymer adsorption the monomer/wall interactions can be strong leading to a pronounced polymer structuring at the wall, whereas far from the wall all the relevant interactions are normally assumed to be weak, and this allows for a universal description of the adsorbed layer. [8]

The structural complexity, however, can become much more rich if just a small fraction of special, strongly-interacting chemical groups (SIGs) are present among the 'passive' steric units in the system. [11] In this Colloquium we consider a number of such systems where, in addition to ordinary weakly-interacting groups, the dissolved unimers (molecules, oligomers or polymers) contain a fraction of SIGs characterized by strong short-range (and possibly directional) pairwise interactions with energy $U_{\text {SIG }} \gtrsim k_{B} T$. These groups can be anisotropic (cf. section 3.2 below), and several distinct types of SIGs can be present in the same molecule (sections 5.2, 5.3). As another example, all aminoacid groups in a peptide molecule can be regarded as SIGs (cf. section 4.3). One of the simplest models of amphiphilic polymers (associative polymers and ionomers [9-11]) involves several isotropic SIGs incorporated in a polymer chain (see the next section). The strong character of the associative interactions can seriously affect the aggregate's structure leading to new morphologies (like formation of disk-like micelles).

\section{From Strong to Super-Strong segregation regime: spherical and nonspherical micelles for copolymers with SIGs}

The concept of SIGs was employed in refs. [9-11] for interpretation of 'multiplets' and 'clusters' in ionomers based on a generalization of the classical theory of blockcopolymer superstructures. The main results of these studies are surveyed below. 
The microphase separation transition in block copolymers is a well-known phenomenon which was studied in detail both theoretically and experimentally [12-16]. For a copolymer built with two distinct types of incompatible chemical units, A and $\mathrm{B}$, tending to avoid each other, the macroscopic phase separation is prevented by the presence of covalent chemical bonds between the blocks. This conflict can be resolved by formation of microdomain structures of various morphologies, related to the so-called microphase separation transition.

Fig. 1a presents the 'classical' phase diagram showing the microphase separation transition and microdomain states in the melt of diblock copolymers (cf. Fig. 1b; for multiblock copolymers the diagram is similar) studied in refs. $[15,16]$ in the framework of self-consistent field approaches. The following variables are used: volume fraction of A-component, $\Phi_{A}$ and the Flory-Huggins interaction parameter $\chi$ between the components $\mathrm{A}$ and $\mathrm{B}$ (multiplied by the total polymerization index $N=N_{A}+N_{B}$, where $N_{A}$ and $N_{B}$ are the numbers of monomer units in the two blocks). The diagram shows that the lamellar phase (Fig. 1d) is characteristic of copolymers with comparable lengths of $A$ - and B-blocks. With an increase of disparity in the block lengths, a cylindrical morphology, where the minor component forms long cylindrical microdomains, emerges first (Fig. 1e), and then a superstructure of spherical micelles formed with minor blocks becomes the most favorable thermodynamically (Fig. 1f).

The critical point corresponds to the microphase separation onset at $\Phi_{A}=$ $0.5, \quad \chi N=(\chi N)_{c}$. In the vicinity of this point the difference between the free energies of all possible phases is not high, and the degree of segregation in the microdomain structure is weak as well. Hence, the conformational statistics of the blocks remain nearly Gaussian. This 'weak segregation regime' was studied theoretically based on the free energy expansion for small concentration fluctuations, [15,17]. By contrast, far from the critical point, at $\chi N \gg(\chi N)_{c}$, the degree of segregation is high, so the microdomains are composed of almost purely $A$ or $B$ units with narrow interfacial layers between $A$ and $B$ regions. This is the 'strong segregation regime' ( $\mathrm{SsR}$ ) whose theoretical description was developed in refs. [14,16,18]. In the $\mathrm{SsR}$ the interfacial tension $\sigma$ between $\mathrm{A}$ and $\mathrm{B}$ domains is high, and the chains are considerably stretched (at least locally) as compared to their Gaussian state. However, the size of a stretched block is still much smaller than its backbone contour length. By comparison, the 'super-strong segregation regime' (SSsR) emerges when $\sigma$ becomes so high (the AB contacts are so expensive energetically) that some blocks tend to reach their maximum allowed elongation degree. [9-11].

As the first example let us consider a melt or a solution of strongly asymmetric block-copolymers where the minor component $\mathrm{A}$ is well segregated from the rest of the system forming dense aggregates (in the $\mathrm{SsR}$ ). ${ }^{1}$ These aggregates are expected to be spherical if

$$
V_{A} \ll V_{B}
$$

where $V_{A}$ and $V_{B}$ are the intrinsic volumes of the respective blocks (cf. Fig. 1a). The number $Q$ of $A$ blocks in each aggregate (dense A-core) is determined

${ }^{1} \mathrm{~A}$ selective solvent which is good for $\mathrm{B}$ but poor for $\mathrm{A}$ is assumed in the solution case. 
geometrically:

$$
Q=\frac{4 \pi}{3} \frac{R^{3}}{V_{A}}
$$

where $R$ is the core radius. The junction points between the blocks are located near the core surface (the A/B interface), while all the blocks are strongly elongated away from the interface, well inside (for $A$-blocks) or well outside it (for $B$-blocks) [14], [16]. Thus, the blocks can be regarded as 'grafted' to the core surface with the grafting density (number of grafting points per unit area)

$$
\frac{1}{s_{\text {intf }}} \equiv \frac{k_{j} Q}{4 \pi R^{2}}=\frac{k_{j} R}{3 V_{A}} \propto Q^{1 / 3}
$$

where $k_{j}=1$ for diblocks, Fig. $1 \mathrm{~b}$, and $k_{j}=2$ (meaning 2 grafting points per block) for multi-block copolymer with large number blocks, Fig. 1c.

The repulsion strength between $A$ - and $B$-blocks can be characterized by the interfacial tension, $\sigma$, which favors larger micelles (with smaller interfacial area per block $s_{\text {intf }} \propto R^{-1}$ ). As a result, the blocks must be highly stretched in the core and (at least partially) in the corona regions, see Fig. 2a. The free energy per micelle in the SsR can be written as a sum of the three terms $[14,16,18]$ :

$$
F=F_{\text {intf }}+F_{\text {core }}+F_{\text {corona }}
$$

where $F_{\text {intf }}=4 \pi \sigma R^{2}$ is the free energy of the interface, $F_{\text {core }}$ is the elastic free energy of A-blocks, and $F_{\text {corona }}$ is the free energy of the B-corona. In the melt case (no solvent) the last term is essentially the conformational elastic free energy of stretched B-blocks in the corona. In the SsR the free energy per block, $F / Q$, is large compared to $k_{B} \mathrm{~T}$. The equilibrium micelle size can be obtained by minimization of $F / Q$ with respect to $Q$.

The stretching energies, $F_{\text {core }} / Q$ and $F_{\text {corona }} / Q$, are increasing with the grafting density $1 / s_{\text {intf }}$ (and hence with the micelle aggregation number $Q$ ), while $F_{\text {intf }} / Q \propto \sigma Q^{-1 / 3}$ is decreasing with micelle size. Obviously, a minimization of $F / Q$, eqs. (4), (2), for the spherical geometry must yield the monotonically increasing equilibrium functions $Q(\sigma)$ and $R(\sigma)$, hence $s_{\text {intf }}$ must decrease as the tension $\sigma$ becomes higher. Therefore, at high enough $\sigma\left(\sigma=\sigma^{*}\right)$ the core size can approach the geometrical maximum corresponding to almost fully stretched A-blocks ( $R \approx L_{A} / k_{j}$, where $L_{A}$ is the A-block contour length) and/or B-blocks near the interface. Concomitantly, the area $s_{\text {intf }}$ decreases and can reach a geometrical minimum corresponding to $R=L_{A} / k_{j}, s_{\min }=3 s_{A}$, where $s_{A}=V_{A} / L_{A}$ is the effective cross-section of an A-block. A further growth of the spherical micelle becomes impossible for $\sigma>\sigma^{*}$. The corresponding new regime (with strongly stretched blocks) was first considered in refs. [9,10]; it is called the 'super-strong segregation' regime, SSsR.

Below we consider in more detail a regular multiblock copolymer case, Fig. 1c (the results for diblock copolymers or telechelic polymers differ only in some numerical prefactors [9-11]). The two criteria of the true SsR mentioned above now read:

$$
R \ll R_{\max } \equiv L_{A} / 2 \quad \text { and } \quad s_{\text {intf }} \gg s_{A B} \equiv \max \left\{s_{A}, s_{B}\right\}
$$


where $s_{B}=V_{B} / L_{B}$ and $L_{B}$ are the cross-section and the contour length of B-blocks. If at least one of these conditions is broken, the system enters the SSsR. Note that $s_{\text {intf }}=3 s_{A}$ for the largest possible $R=L_{A} / 2$, cf. eq. (3) (note that for $R>L_{A} / 2$ none of the A-blocks can reach the micelle center), hence typically the second condition is weaker.

For $R \ll L_{A} / 2$ (in the $\mathrm{SsR}$ ) the stretched A-blocks are in the linear elasticity regime, so the core elastic energy is

$$
\frac{F_{\text {core }}}{k_{B} T Q} \simeq k_{\text {core }} \frac{R^{2}}{L_{A} l_{A}}
$$

where $k_{\text {core }}=3 \pi^{2} / 20$ and $l_{A}, l_{B}$ are the Kuhn segments of A, B blocks (it is also assumed that $\left.L_{A} \gg l_{A}, L_{B} \gg l_{B}\right)$. [16] In the block-copolymer melt the B-blocks are mostly stretched near the interface if the core is small compared to the corona (i.e., for the asymmetric case, condition (1)), and their elastic energy can be expressed using the electrostatic analogy [16]:

$$
\frac{F_{\text {corona }}}{k_{B} T} \simeq k_{\text {corona }} \frac{Q_{\text {eff }}^{2}}{2 R}, \quad k_{\text {corona }}=\frac{3}{4 \pi} \frac{s_{B}}{l_{B}}
$$

where $Q_{\text {eff }}=k_{j} Q=2 Q$ in the case of multi-block-copolymer melt. As a result

$$
\frac{F_{\text {core }}}{F_{\text {corona }}} \simeq \frac{3 \pi^{2}}{40}\left(\frac{s_{A} l_{B}}{s_{B} l_{A}}\right) \quad(\mathrm{SsR}, \text { melt })
$$

for a spherical core in the melt. Note that both $F_{\text {core }} / Q$ and $F_{\text {corona }} / Q$ are proportional to $Q^{2 / 3}$, hence they significantly increase with $Q$.

Turning now to the block-copolymer solution in a selective solvent which is good for $B$-blocks, but is non-solvent for A-blocks, the well-known scaling approach can be applied to get the corona free energy in this case [23-25]. The equilibrium corona structure is determined by a balance between the free energy of 'elastically' stretched B-blocks on the one hand, and excluded volume interactions of B-units in the solvent on the other hand (the latter interactions tend to swell the corona while the stretching limits the degree of swelling). This balance leads to the Daoud-Cotton scaling picture of blobs of variable sizes, the energy of the corona being simply proportional to the total number of blobs:

$$
\frac{F_{\text {corona }}}{k_{B} T} \sim Q^{3 / 2} \ln \left(\frac{R_{2}}{R}\right)
$$

where $R_{2} \sim Q^{(1-\nu) / 2} l_{B}\left(L_{B} / l_{B}\right)^{\nu}\left(s_{B} / l_{B}^{2}\right)^{\nu-1 / 2}$ is the external radius of the corona, $\nu \simeq 0.59 \simeq 3 / 5$ is the Flory exponent for a flexible coil in a good solvent. Again, $F_{\text {corona }} / Q$ increases with $Q$ (being roughly proportional to $Q^{1 / 2}$ ).

Minimization of the free energy per block, $F / Q$, cf. eq. (4), gives the equilibrium radius $R$ of the micelle core. For the melt case it gives $[9,10]$ :

$$
R=\left[\frac{3}{4} \frac{\sigma}{k_{B} T} \frac{l_{B}}{s_{B}}\left(L_{A} s_{A}\right)^{2} \Theta^{-1}\right]^{1 / 3}, \quad \Theta \equiv\left(1+\frac{3 \pi^{2}}{40} \frac{s_{A} l_{B}}{s_{B} l_{A}}\right) \quad \text { (SsR, melt) }
$$

hence, the radius $R \propto \sigma^{1 / 3}$ is an increasing function of $\sigma$. For the case of selective solvent in the SsR, the term $F_{\text {core }}$ is always smaller than $F_{\text {corona }}$, cf. eqs. (6), (9): 


$$
\frac{F_{\text {core }}}{F_{\text {corona }}} \sim Q^{1 / 6}\left(\frac{s_{A}^{2}}{L_{A} l_{A}^{3}}\right)^{1 / 3} \sim\left(\frac{R}{L_{A}} \frac{s_{A}}{l_{A}^{2}}\right)^{1 / 2} \ll 1 \quad \text { (SsR, solution) }
$$

and the minimization of $F / Q$ leads to the scaling result

$$
R \sim\left(\frac{\sigma}{k_{B} T}\right)^{2 / 5}\left(L_{A} s_{A}\right)^{3 / 5} \quad(\text { SsR, solution })
$$

hence $R \propto \sigma^{2 / 5}$ shows an unbounded increase with $\sigma$. Thus, the limits of the transition to the SSsR, eqs. (5), can be approached as the surface tension $\sigma$ increases. Once the system enters the SSsR, the spherical micelles cannot grow any more, so the term $F_{\text {intf }}=2 Q s_{\text {intf }} \sigma$ begins to dominate the total free energy (4).

To further consider the SSsR, let us assume for simplicity that A-blocks are thicker and more rigid than B-blocks:

$$
s_{A} \gg s_{B}, \quad l_{A} / s_{A} \gg l_{B} / s_{B}
$$

(a more general situation was considered in ref. [11]). Hence $F_{\text {core }} \ll F_{\text {corona }}$ both for melt and solution cases (cf. eqs. (8), (11) and note that $l_{A}^{2} / s_{A} \gg l_{B}^{2} / s_{B} \gtrsim 1$ ), so eqs. (10), (12) are asymptotically exact for the whole SsR region (and in the SsR to SSsR transition region). Noteworthily, the B-blocks are far from complete stretching even if A-blocks are completely stretched since $s_{B} \ll s_{A}$.

At some large enough tension, $\sigma=\sigma^{*}$, the core radius $R$ (according to eqs. (10), (12)) reaches the value $R_{\max } \equiv L_{A} / 2$ (the half-length of a completely stretched A block, cf. eq. (5)). It is important to note that even if the core radius, $R$, is close to the limiting value $R_{\max }$, our expressions for the corona and interface free energies remain valid. Therefore the predictions, eqs. (10), (12) are quantitatively valid in the whole region $\sigma_{\min } \ll \sigma<\sigma^{*}$, where $\sigma^{*}$ is determined by the condition $R\left(\sigma^{*}\right)=R_{\max } \quad$ (note that the condition $s_{\text {intf }}>s_{A B}$ is never violated since $\left.s_{B} \ll s_{A}\right) \quad[9,10]$ :

$$
\frac{\sigma^{*}}{k_{B} T}=\frac{1}{6} \frac{L_{A} s_{B}}{l_{B} s_{A}^{2}} \quad(\text { for melt }) ; \frac{\sigma^{*}}{k_{B} T} \sim \frac{L_{A}}{s_{A}^{3 / 2}}(\text { for solvent })
$$

Above $\sigma^{*}$ the micelle core cannot grow further while retaining the spherical shape as this growth would imply creation of a very unfavorable empty hole in its central region (hence, eqs. (10), (12) are not valid any more), so the interfacial energy per block cannot be decreased this way. It is still possible to decrease this energy? The answer is positive: the free energy decrease can be achieved by changing the micelle shape. Indeed, the formal geometrical restriction in the SSsR is that the minimum distance between any point inside the core and its surface must not exceed $R_{\max }$. The aggregates can still grow in one or two dimensions while obeying this restriction: in the first case they tend to become cylinders of radius $R_{\max }$ (Fig. 2b), in the second - disks of thickness $H=2 R_{\max }=L_{A}$ (Fig. 2c). In any case the micelles tend to become non-spherical at $\sigma>\sigma^{*}$. In fact, the specific area per grafting point, $s_{\text {intf }}=S_{\text {intf }} /(2 Q)$, is determined solely by the geometry of the micellar core (note that $2 Q$ is the number of junction points on the core surface). For the spherical shape $s_{\text {intf }}^{(\mathrm{sph})} \simeq 3 s_{A}$ (see eq. (3) with $R=R_{\max }$ ), for long cylinders $s_{\text {intf }}^{\text {(cyl) }} \simeq L_{A} s_{A} / R_{\max }=2 s_{A}$, while for wide disks $s_{\text {intf }}^{\text {(disk) }} \simeq s_{A}$. For large $\sigma$ the surface energy term, $F_{\text {intf }}=2 Q s_{\text {intf }} \sigma$, becomes dominant 
in the sum (4), hence the wining morphology must minimize the specific area $s_{\text {intf }}$ since $F_{\text {intf }} / Q \propto s_{\text {intf. }}$ This argument points to the disk-like shape for $\sigma \gg \sigma^{*}$.

The transition from spherical to disk-like aggregates was considered in ref. [10]. The equilibrium shape of the micelle is determined by minimization of the free energy per A-block which is close to $\left(F_{\text {intf }}+F_{\text {corona }}\right) / Q$ since $F_{\text {core }} \ll F_{\text {corona }}$ also for $\sigma>\sigma^{*}$ (cf. eqs. (1), (13)). Two alternative shapes minimizing the core surface energy were analyzed [10] with the additional condition that no inner point of the core is located farther than $R_{\max }$ from the surface: (i) a spherocylinder, Fig. 2b, and (ii) a disk with a rounded edge, Fig. 2c. Both shapes can be obtained by a rotation of a rectangular of height $H=2 R_{\max }$ and width $D-H$ with two half-circles of diameter $H$ adjoined its sides around the vertical (to get a disk) or horizontal (cylinder) symmetry axis. It was demonstrated [10] that for $\sigma>\sigma^{*}$ the free energy minimum always corresponds to an oblate (disk-like) shape, while prolate (spherocylinder) aggregates are never selected thermodynamically for strongly asymmetric block-copolymers (cf. eq. (1)). The transition at $\sigma=\sigma^{*}$ is continuous (of the second-order type). As the thickness of the corona $R_{2}$ strongly exceeds $R_{\max }$ (cf. Fig. 2d), there is a range of the core diameter, $H<D \ll R_{2}$, where the corona is still nearly spherical while the core is non-spherical. Minimization of $\left(F_{\text {intf }}+F_{\text {corona }}\right) / Q$ gives us the equilibrium size of the disk-like micelle (cf. ref. [10]):

$$
D \simeq H+\frac{2}{3} H\left(\left(\sigma / \sigma^{*}\right)^{1 / 2}-1\right)(\text { for melt }) ; D \sim L_{A}\left(\sigma / \sigma^{*}\right)^{1 / 2}(\text { for solution })
$$

In both cases $D \propto \sigma^{1 / 2}$ and $D \propto L_{A}^{1 / 2}$ in the limit $\sigma \gg \sigma^{*}$.

Thus, the micelle core becomes larger as the surface tension $\sigma$ increases. Beyond some threshold tension, for $\sigma>\sigma^{* *}$, the edge contribution to the interfacial free energy gets so unfavorable that the diameter $D$ of the most stable disk-shaped micelle becomes infinite. The transition occurs when the disk diameter $D$ becomes comparable with the characteristic thickness of the corona $R_{2}$. For the case of solution the blob picture yields $R_{2} \sim l_{B}\left(L_{B} / l_{B}\right)^{\nu}\left(s_{B} / l_{B}^{2}\right)^{\nu-1 / 2} Q^{(1-\nu) / 2}$ in the regime $R_{2} \lesssim D$, while for the melt the incompressibility condition gives $R_{2}^{3} \sim Q L_{B} s_{B}$. Hence [10]

$$
\sigma^{* *} \simeq \frac{3}{2 \pi} \frac{s_{B}^{3}}{s_{A}^{4}} \frac{L_{B}^{2}}{L_{A} l_{B}}(\text { for melt }) ; \sigma^{* *} \sim\left(\frac{s_{B}}{s_{A}}\right)^{1 / \nu} \frac{L_{B}^{2}}{L_{A} l_{B}^{2} s_{A}^{1 / 2}}(\text { for solution })
$$

Note that in both cases $\sigma^{* *} \propto L_{B}^{2} / L_{A}$, whereas $\sigma^{*} \propto L_{A}$, see eqs. (14), (16). Hence, for highly asymmetric block-copolymers (cf. the condition (1)) the region of stability of disk-like multiplets is very wide since $\sigma^{* *} / \sigma^{*} \propto\left(L_{B} / L_{A}\right)^{2} \gg 1$. Thus, two sequential transitions take place as the core surface tension $\sigma$ increases: first from spherical to disk-like multiplets, and then from disks to infinite lamellar sheets.

A more detailed analysis [10] shows that usually the transition from spherical to disk-like aggregates is of the second order, while the transition from disks to lamellae is of the first order. On the other hand, it was shown numerically, for both melts and solutions, that cylindrical micelles are always less stable than disks in the SSsR for copolymer compositions corresponding to spherical micelles in the ordinary SsR. Moreover, for the model considered above (in the melt case) we established that the cylindrical microstructure is never stable thermodynamically also in the standard SsR. This difference from the classical behavior (cf. Fig. 1a) 
is due to strong asymmetry in size/rigidity of $\mathrm{A}$ and $\mathrm{B}$ units (cf. eq. (13)); it also leads to a wider regime of stability for the spherical microstructure extending from $x=x_{A} \ll 1$ to $x \approx 3$, where $x=V_{A} / V_{B}=\Phi_{A} /\left(1-\Phi_{A}\right)$ is the copolymer composition parameter and $x_{A} \sim l_{A} s_{A} / V_{B}$ (note that $x \sim x_{A} L_{A} / l_{A}$ ). The eventual diagram of the predicted equilibrium morphologies of microdomains formed by the minor component $\mathrm{A}$ is shown in Fig. 3. In the case of a very dilute block-copolymer solution in the 'lamellar' regime $\left(\sigma>\sigma^{* *}\right)$ the copolymers may form 'unilamellar vesicles' (Fig. 2d) with one or several chains per vesicle depending on concentration. Thus, strong attraction of minor A-units gives rise to a qualitatively new regime of 'super-strong segregation' and new microdomain morphologies (cp. phase diagrams, Fig. 1a and Fig. 3). This effect demonstrates how quantative changes in physical parameters of a system can result in qualitative changes of its structural behavior.

Since $\sigma^{*}$ is proportional to $L_{A}$ (cf. eqs. (14)), the superstrong segregation regime is usually not met in ordinary block copolymer systems. However this regime is characteristic for ionomers and associating polymers since the core-surface tension, $\sigma$, is typically large for these systems, while $L_{A}$ (which is proportional to the size of an ionic or associating group) is relatively small. The disk-like morphology was found in computer simulation studies of solutions of strongly associating copolymers [26,27]. Turning to experimental data, the disk-like [28-30], lamellar [30] and other micellar morphologies have been reported for ionomeric and amphiphilic block-copolymer systems [32]. In particular, similar structures have been observed in polyethylene-containing block-copolymers in decane and water [33,34], in polypeptide diblock-copolymers [35] and in ionic complexes of $\mathrm{AB}$ and $\mathrm{CD}$ copolymers (disk-shaped Janus micelles with coacervate core and water-swollen corona) [36]. An interesting phenomenon has been observed in a methacrylate ionomer melt: as ionic content increases, the ion-rich microstructure transforms from a continuous to a discontinuous one [31]. This effect can be explained in the framework of the SSsR theory: an increase of the ionic fraction implies a slight increase of the average size of ionic blocks $\left(L_{A}\right)$, thus $\sigma^{*}$ must increase as well triggering a transition from disk-like micelles, which can easily form a quasicontinuous phase, to disconnected spherical micelles.

\section{Geometrical mismatch and frustration effects in systems with SIGs}

Geometrical frustration in condensed matter physics refers to situations where a local pattern of molecular packing cannot propagate over the whole volume of the system [38,37] since any global periodic structure implies significant deformations of certain rigid fragments of interacting particles. Being strongly mutually attractive and rigid, the SIGs are prone to beget frustrations of various kinds, including size mismatch, orientational and topological frustrations. In this section we discuss the first frustration type arising when sizes of two (or more) molecular elements contradict each other precluding formation of a structure with optimally saturated physical bonds. If such a system adopts a uniform amorphous structure, it always leads to a free energy penalty due to a distributed mismatch. Alternatively, the molecules can arrange in domains of finite size (microphase structuring), so that the structure inside the domains is nearly perfect (energy minimizing), while the 
domain interfaces imply an extra energy cost.

\subsection{The tilting transition: head-rod model}

We start our consideration with transitions in flat-layer molecular membranes which are useful for engineering of functional materials. [52] Noteworthily, nature also uses the membrane self-assembly to construct functional microstructures for living beings. In particular, it is well-known that lipid molecules (involving watersoluble 'heads' jointed with hydrophobic tails) can form bilayer structures [51], which have been studied for a long time and can serve as models for biological membranes. [39]

A simple but interesting consequence of a geometrical mismatch in layered structures of amphiphilic molecules is the tilting effect observed, for example, in Langmuir monolayers [40] and in phospholipid bilayer membranes showing a $L_{\beta} \rightarrow L_{\beta^{\prime}}$ transition. [102,103] In the case of multilayer smectic structures the molecular tilting is associated with the transition between smectic $A$ and smectic $C$ mesophases $(\operatorname{Sm} A \rightarrow \operatorname{Sm} C)$ [5].

As an illustration of this effect, let us consider a simple model of a flat layer formed by amphiphilic head-rod unimers with bulky solvophilic spherical 'head' groups (with specific area $s_{h}$ per head) and stiff solvophobic rod blocks of length $L_{r}$ with specific cross-section area $s_{r}$, Fig. 4a. Assuming a perfect orientational order of the stiff blocks, one can write the free energy of the layer (per unimer) as:

$$
F=F_{\text {head }}(\psi)+F_{\text {rod }}(\psi)
$$

where $\psi$ is the angle between the director of the rod orientation and the normal vector to the layer, cf. Fig. 4c, $F_{\text {head }}(\psi)$ is the energy cost for the head rotation (which can disrupt the sublayer of heap groups), $F_{\text {rod }}=V_{1} f_{\text {rod }}$, where $V_{1}=s_{h} L_{r} \cos \psi$ is the volume per molecular unit in the solvophobic sublayer, and $f_{\text {rod }}$ is the free energy per unit volume in this sublayer. In the spirit of the mean-field FloryHuggins model [3] the mixing free energy of the rod sublayer is $f_{\text {rod }}=f_{\text {id }}+f_{\text {mix }}$, where $f_{\text {id }}$ is coming from the ideal-gas entropy of solvent molecules, and $f_{\text {mix }}$ is the enthalpic contribution due to solvent/rod interactions:

$$
f_{\text {id }}=(1-\phi) \ln (1-\phi) k_{B} T / v_{s}, \quad f_{\text {mix }}=\chi \phi(1-\phi) k_{B} T / v_{s}
$$

where $v_{s}$ is the volume of a solvent molecule, $\chi$ is the Flory-Huggins interaction parameter, and $\phi$ is the volume fraction of rods in the sublayer:

$$
\phi \equiv s_{r} L_{r} / V_{1}=\left(s_{r} / s_{h}\right) / \cos \psi
$$

For $\psi=0$ (no tilt, $\operatorname{Sm} A$ state, Fig. $4 b$ ) the concentration is minimal, $\phi=\phi_{\min }$, where

$$
\phi_{\min } \equiv s_{r} / s_{h}
$$

is related to the area mismatch. Note that $\phi_{\min } \leq \phi \leq 1$ and correspondingly $0 \leq \psi \leq \psi_{\max }$, where $\cos \psi_{\max }=s_{r} / s_{h}$. The equilibrium state of the layer can be obtained by minimization of the total free energy, eq. (17). 
Let us consider first the simplest case when $F_{\text {head }}$ is not affected by the rod rotation: $F_{\text {head }}(\psi) \equiv$ const. Then, the optimal tilt angle $\psi$ corresponds the minimum of $F_{\text {rod }}$ with respect to $\phi$ (once $\psi$ is expressed in terms of $\phi$ using eq. (19)). Thus we get

$$
\frac{\mathrm{d} F_{\text {rod }}}{\mathrm{d} \phi}=K\left(\tilde{f}_{\mathrm{id}}(\phi)-\chi\right), \quad \tilde{f}_{\mathrm{id}}(\phi) \equiv-\frac{\ln (1-\phi)}{\phi^{2}}-\frac{1}{\phi} \quad K \equiv \frac{k_{B} T s_{r} L_{r}}{v_{s}}
$$

where the function $\tilde{f}_{\text {id }}(\phi)$ is an increasing positive function of $\phi, \tilde{f}_{\text {id }}(0)=0.5$, $\tilde{f}_{\text {id }}(1)=+\infty$. If the solvent is moderately poor, $\chi \leq \chi^{*}, F_{\text {rod }}$ is always increasing with $\psi$, hence the membrane stays in the original symmetric state $\left(\psi=\psi_{A} \equiv 0\right.$, $\left.\phi=\phi_{A} \equiv \phi_{\min }\right)$. Here the critical interaction parameter is

$$
\chi^{*}=\tilde{f}_{\text {id }}\left(\phi_{\min }\right)
$$

If the rod insolubility exceeds the critical value, $\chi>\chi^{*}$, the equilibrium layer state is tilted (the $\mathrm{Sm} C$ phase) with $\psi=\psi_{C}>0$ and $\phi=\phi_{C}>\phi_{\min }$. The parameters $\psi_{C}, \phi_{C}$ are determined by equations:

$$
\tilde{f}_{\mathrm{id}}\left(\phi_{C}\right)=\chi, \quad \psi_{C}=\arccos \left(\phi_{\min } / \phi_{C}\right)
$$

Thus, as $\chi$ is increased, a second order phase transition from $\operatorname{Sm} A$ to $\operatorname{Sm} C$ tilted state takes place at $\chi=\chi^{*}$. For $\chi>\chi^{*}: \phi_{C}-\phi_{\min } \propto \chi-\chi^{*}, \psi_{C} \propto\left(\chi-\chi^{*}\right)^{\mathbf{0 . 5}}$, $F\left(\phi_{\min }\right)-F\left(\phi_{C}\right) \propto\left(\chi-\chi^{*}\right)^{2}$.

If the head rotation implies an energy penalty, $F_{\text {head }}(\psi)=k_{h} \psi^{2} / 2$ for small $\psi$, and $k_{h}$ is below some threshold:

$$
k_{h}<k_{* *}, \quad k_{* *}=\left.\frac{3 K}{5}\left[2 \frac{\ln (1-\phi)}{\phi^{3}}+\frac{2-\phi}{\phi^{2}(1-\phi)}\right]\right|_{\phi=\phi_{\text {min }}}
$$

the transition to the tilted state shifts to $\chi_{A C}=\chi^{*}+k_{h} /\left(K \phi_{\min }\right)$, while the transition nature remains the same (of the second order). For larger $k_{h}, k_{h}>k_{* *}$, a discontinuous first order $A-C$ phase transition is predicted.

\subsection{Rod-coil mesophases}

The model of head-rod surfactant considered above (cf. Fig. 4a) involves two types of SIGs: the heads forming the hydrophilic sublayer with fixed area per head, and the rods arranged densely and parallel to each other. A similar behavior (including the $\operatorname{Sm} A \rightarrow \operatorname{Sm} C$ transition) was predicted for a melt of rodcoil copolymers (Fig. 5a) involving just one type of SIGs (rods). [41] The rodlike SIGs are supposed to be perfectly aligned as in the head-rod model considered above. The flexible tail parts tend to separate from rods, and this repulsion is characterized by the standard $\chi$-parameter (which now accounts for the rod/tail rather than $\mathrm{rod} / \mathrm{solvent}$ interactions $): f_{\text {mix }}=\chi \phi(1-\phi) k_{B} T / v_{t}$, where $v_{t}$ is the volume per monomer in a flexible tail. For low incompatibility $\chi$, the copolymers are distributed homogeneously in space forming a nematic phase $(\mathrm{N})$, Fig. 5b. With an increase of the $\chi$-repulsion, the system experiences a sequence of phase transitions between N-phase and various types of smectic phases illustrated in Fig. 5c-f. The phase diagram (drawn according to ref. [41]) is shown in Fig. 6. There are two 
distinct types of smectic phases formed in this system: phases $A_{1}$ and $C_{1}$ involve single smectic layers, where each lamellar layer of rods is formed as a mixture of interdigitated oppositely directed rods, Fig. 5c,e. By comparison, the phases $A_{2}$ and $C_{2}$ show double smectic layers where rigid fragments form sublayers of co-directed rods, and sublayers with opposite orientations of rods are combined in pairs (double layers) surrounded with 'brushes' of flexible blocks, Fig. 5d,f. In smectics $A$ the rods are perpendicular to the layers, while in smectics $C$ they are tilted with some angle $\psi \neq 0$.

Noteworthily, these smectic phases are similar to the classical lamellar microdomain structures of AB block-copolymers with flexible blocks $[15,16]$ (cf. Fig. 1a) where the copolymer components form parallel sublayers, and each flexible block is extended away from an A/B interface. In the melt of rod-coil molecules, the tails also separate from the rods for large $\chi$ beyond some threshold $\chi_{\mathrm{N}-\mathrm{Sm}}$ depending on the molecular composition parameter $\lambda \equiv V_{t} / V_{r}$, where $V_{t} \equiv L_{t} s_{t}$ is the total volume of a flexible tail, and $V_{r} \equiv L_{r} s_{r}$ is the volume of a rod block (cf. Fig. 5a). The diagram of Fig. 6 was obtained [41] for the regime of 'slim' tails, $s_{t} \lesssim s_{r}, l_{t} / s_{t} \ll L_{r} / s_{r}$, where $l_{t}$ is the Kuhn segment length of the flexible block.

The critical point for the $\mathrm{N} \rightarrow \mathrm{Sm}$ transition (cf. Fig. 6) corresponds to $\lambda^{\mathrm{cr}} \simeq 1$ and $\chi_{\mathrm{N}-\mathrm{Sm}}^{\mathrm{cr}} \simeq v_{t} / V_{r}$; the critical volume composition is thus nearly symmetric, while the critical interaction parameter is inversely proportional to the copolymer contour length in harmony with the melt behavior of classical flexible block-copolymers [15] (cf. Fig. 1a). For the case of rod-coil molecules with the dominant rod part, $\lambda<1$, the system shows a second order transition to the $\operatorname{Sm} A_{1}$ phase at $\chi=\chi_{1}$. For the case of dominant tails, $\lambda>1$, the system undergoes a first order transition from nematic directly to $\mathrm{Sm} C_{1}$ phase at $\chi=\chi_{4}\left(\chi_{1}, \chi_{4}\right.$ are defined in the caption of Fig. 6).

In the diagram Fig. 6 the tilted $\mathrm{Sm} C$ phases generally occupy the regions to the right of the more symmetric $\operatorname{Sm} A$ phases, that is, the regions with longer flexible tails (higher $\lambda$; see the lines (8) and $(6)+(9)$ in Fig. 6). The reason is simply that an increase of the tilt angle $\psi$ reduces the elastic energy cost for the tail stretching just as the finite disk formation does this for systems of amphiphilic block-copolymer molecules with SIGs considered in section 2 (cf. eq. (15)). However, as a result of the interfacial area increase, the energy of interfaces between the rod and the tail sublayers increases as well. The interfacial surface energy is proportional to $\propto \chi^{0.5}$, hence it becomes more important at high $\chi$ 's and finally causes a decrease of the tilt angle. It leads eventually to a transition to the state with $\psi=0$ occurring at the lines (6) and (8) which show the second order transitions $\mathrm{Sm}_{1} \rightarrow \mathrm{Sm} A_{1}$ and $\mathrm{Sm}_{2} \rightarrow \mathrm{Sm} A_{2}$, respectively (cf. Fig. 6). On the other hand, the doubling of the smectic layers takes place at the lines (2) and (7) (transitions $\mathrm{Sm} A_{1} \rightarrow \mathrm{Sm} A_{2}$ and $\mathrm{Sm} A_{1} \rightarrow \mathrm{Sm} C_{2}$, respectively). The doubling occurs as a strong first order transition. The total rod/coil interfacial area is obviously smaller (by a factor of $\sim 2$ ) in the double-layer smectic phases (as compared to single-layer smectics), hence the double-layer structures are observed for higher interaction parameters $\chi$ (with higher interfacial tension). By contrast, the single smectics are selected at lower $\chi$ because of their higher entropy (the rods with opposite orientations are not separated there). 


\subsection{Frustration-defined patterns}

Let us now turn to more complicated situations where size/area mismatches cannot be resolved just by tilting, so some sort of pattern (microstructure) must be formed to heal the mismatch. A relevant example is provided by a flat selfassembling (SA) layer of dumbbell-like A-B-A molecules consisting of 3 SIGs (one B-SIG in the middle and 2 A-SIGs at the ends) connected with 2 soluble spacers, Fig. 7a. All SIGs strongly repel other components (the opposite SIGs, the spacers and the solvent); SIGs of each type tend to form a separate 2-dimensional (2D) layer with the specific area $s_{i}$ per SIG $(i=A, B)$. In the general case, there is an area mismatch between $\mathrm{A}$ - and B-layers of SIGs, say $s_{A}<s_{B}$. Such geometrical frustration of the whole layer structure [43] can result in splitting of the minor SIG A-sublayer, i.e. in pattern formation, as explained below.

In a homogeneous layer of A-B-A molecules (Fig. 7b) the area per SIG must be close to $s_{B}$. On the other hand, the A-SIGs prefer a denser packing (with smaller area per SIG). As a result, the minor sublayers $A$ may tend to split into ribbons (Fig. $7 \mathrm{c}$ ). Let us choose the $x$-coordinate axis perpendicular to the ribbons in the layer plane. The ribbon width $W$ can be obtained by minimization of the specific free energy (per unit area of the layer):

$$
F / 2=\frac{2 \gamma}{W}+\frac{k_{A}}{2 W} \int_{-W / 2}^{W / 2} \varepsilon^{2}(x) \mathrm{d} x+\frac{k_{\mathrm{s}}}{2 W} \int_{-W / 2}^{W / 2}(\tilde{x}-x)^{2} \mathrm{~d} x
$$

where $\gamma$ is the A-layer edge tension, $k_{A}$ is the elastic modulus for stretching of an $A$-sublayer and $k_{\mathrm{s}}$ is the normalized shear elasticity constant of the spacers. For simplicity, the $B$-sublayer is assumed to be much more rigid than $A$-sublayer, hence the positions of SIGs within the $B$-sublayer are fixed. The function $\tilde{x}(x)$ gives the current position of an $A$-SIG connected with a $B$-SIG having the coordinate $x$, and $\varepsilon(x)=\mathrm{d} \tilde{x} / \mathrm{d} x-\left(1-\varepsilon_{0}\right)$ is the local $x$-strain of the $A$-sublayer with respect to its ground state; here $\varepsilon_{0} \equiv 1-s_{A} / s_{B}$ is the degree of area mismatch. The standard minimization of the functional (25) with respect to the profile $\tilde{x}(x)$ gives the following specific free energy for ribbons of width $W$ :

$$
F(W)=\frac{2 \gamma}{W}+k_{A} \varepsilon_{0}^{2}\left(\frac{1}{2}-\frac{\tanh (q W / 2)}{q W}\right), \quad q \equiv\left(\frac{k_{\mathrm{s}}}{k_{A}}\right)^{1 / 2}
$$

If the mismatch degree $\epsilon_{0}$ is small enough (alternatively, if the edge energy is large),

$$
\gamma \geq \gamma_{\mathrm{cr}} \equiv \frac{k_{A} \varepsilon_{0}^{2}}{2 q}=\frac{\varepsilon_{0}^{2}}{2 q}\left(\frac{k_{A}^{3}}{k_{\mathrm{s}}}\right)^{1 / 2}
$$

the optimal configuration of the layer is homogeneous (no pattern): $W=\infty$, $\widetilde{x}(x)=x, \varepsilon(x)=\varepsilon_{0}$. In this case the spacers (s) are not deformed and the elastic energy penalty is solely due to the $A$-sublayer: $F=F_{0} \equiv k_{A} \varepsilon_{0}^{2} / 2$. However, if $\gamma_{\mathrm{cr}}>\gamma$, the layer splits into ribbons which optimal width defined by the condition:

$$
\frac{2 q \gamma}{k_{A} \varepsilon_{0}^{2}}=\frac{\sinh (q W)-q W}{\cosh (q W)+1}
$$

The equilibrium ribbon width $W_{*}$ is: 


$$
W_{*} \simeq\left\{\begin{array}{cc}
-q^{-1} \ln \delta, & \text { if } \delta \equiv 1-\gamma / \gamma_{\mathrm{cr}} \ll 1 ; \\
\left(24 \gamma / k_{\mathrm{s}} \varepsilon_{0}^{2}\right)^{1 / 3}, & \text { if } \gamma_{\mathrm{cr}} \gg \gamma
\end{array}\right.
$$

The pattern formation at $\gamma=\gamma_{\text {cr }}$ is a weak first order transition with $\Delta F \sim-\delta / \ln \delta$ for $\delta \equiv 1-\gamma / \gamma_{\text {cr }} \ll 1$ (near the transition point), hence a hysteresis may be expected in practice. A further analysis shows that more complicated $2 \mathrm{D}$ patterns (involving circular or hexagonal domains) are less favorable thermodynamically than the pattern of ribbons considered above.

Amphiphilic molecules of roughly A-B-A architecture have been studies in ref. [43]. The molecular structure is shown in Fig. 15a,b; its main parts are (see section 5.2 for details): amide groups ('A', red), aromatic rings ('B', green) and hydrocarbon spacers (blue). It was found [43] that such BHPB10 molecules self-assemble in cyclohexanone solution forming interdigitated double layers which involve 1 aromatic sublayer and 2 sublayers of amide groups. In this structure the amide sublayers are split producing a pattern of nanoribbons (Fig. $7 \mathrm{~d}$ ) which are similar to the ribbons shown in Fig. 7c. It is important that the multiple H-bonds between amide groups form parallel physical chains. As a result, the ribbon edge tension is strongly anisotropic with a pronounced minimum for the edge orientation along the chains of H-bonds (as any other orientation demands cutting these chains at the edge).

A similar pattern formation is expected also in amphiphilic molecular layers adsorbed on a solid support. In this case a minimalist model involves just dumbbell molecules A-B, $s_{A}<s_{B}$, with adsorbed B-SIGs leading to a frustration-defined stripes pattern of A-SIGs (Fig. 7e). From a broader perspective, surface cracking patterns are widespread in physical systems involving two distinct interconnected layers on a rigid support with area mismatch between the layers. The pattern formation is often due to a drying of the upper layer. Usual examples include desiccating cracking patterns formed on a mud and on old paintings or ceramic glaze (the so called 'craquelure') [42]. Here the upper surface layer is loosing a liquid (plastifier) more rapidly, so it shrinks in volume and becomes less flexible, while the layer underneath is less dry and ensures elastic connection to the support.

\subsection{Macromolecular helicity stemming from geometrical frustration}

Another example of geometrical frustration effects is provided by comb-like macromolecules (molecular bottle-brushes). [45,46] As a simple toy-model let us consider a linear backbone with bulky side groups connected to it with rigid spacers. The contour length per repeat unit is $b$, while the effective diameter of side groups is $B>b$, Fig. $8 \mathrm{a}$. In order to ease the strong repulsion between the side groups, they have to rotate twisting the chain by an angle $\psi$ at each repeat unit (Fig. 8b). As a result, the distance between the neighbouring side-groups increases to $R=\left(b^{2}+2 r^{2}(1-\cos \psi)\right)^{1 / 2}$. The equilibrium angle $\psi$ can be obtained by minimization of the corresponding free energy per unit:

$$
F=\frac{k_{\mathrm{tw}}}{2} \psi^{2}+\frac{k_{\mathrm{sg}}}{2}(B-R)^{2}
$$

Here $k_{\mathrm{tw}}$ is the main chain twisting elastic modulus and $k_{\mathrm{sg}}$ is the effective modulus of elastic repulsion between the side groups. For weak repulsions $k_{\mathrm{sg}} \leq k_{\mathrm{sg}}^{*}=$ 
$k_{\mathrm{tw}}\left(r^{2}(B / b-1)\right)^{-1}$, the optimal chain configuration in dilute solution corresponds to $\psi=0$ (no twist). By contrast, a twisted macromolecular configuration with $\psi \neq 0$ is predicted at larger $k_{\mathrm{sg}}>k_{\mathrm{sg}}^{*}$. If there is a strong coupling between rotation angles at two consequent repeat units making the rotation in the same direction more favorable (with an energy penalty $F_{\mathrm{pe}}$ for changing the rotation direction; this penalty may originate, for example, from a non-spherical shape of side groups, cf. Fig. 8c), the comb-like chain will tend to form long helices if $k_{\mathrm{sg}}>k_{\mathrm{sg}}^{*}$ and $F_{\mathrm{pe}} \gg k_{B} T$ (Fig. 8d). In this case the typical length of one helical fragment is proportional to $\exp \left(F_{\mathrm{pe}} / k_{B} T\right)$ (in analogy with the coil-helix transition in a homopolymer chain [3]).

Interestingly, such spontaneous local molecular helicity was observed in solutions of many achiral polymers with rigid enough main chains provided that the interactions between the side groups are significant [47]. Sometimes the initial helical configuration is virtually 'frozen' at room temperatures. This happens, e.g., in solutions of polyacetylenes having formally achiral chains (no chiral centers) whose segments, however, show a weak but permanent spontaneous local twist. If their polymerization takes place in a cholesteric reaction bath, a so-called 'helical polyacetylene' can be formed with permanent memory of the original co-directed twists even after the cholesteric solvent is completely washed out with toluene $[48,49]$. In other cases the helicity is 'dynamical': the points of helix reversals can readily move along the polymer chain at room temperature. However, the average length of a chiral helical sequence can still be very long, thus making such polymers capable to switch their helicity in response to a tiny amount of a chiral additive acting thus as a 'helicity amplifier'. The latter type of behavior was demonstrated with some polyvinyls and polyisocyanates $[50,47]$.

\section{Systems with chiral SIGs}

So far we discussed the structure formation driven by area mismatch between the SIGs involved in amphiphilic molecules (head/tail in phospholipids, backbone/side-groups in comb-like polymers, etc.). Here we turn to self-assembling (SA) morphologies emerging in the presence of chiral entities in the system. We start with surfactant bilayers that often form cylindrical and helical structures instead of flat layers.

\subsection{Landau-type phenomenological theories of SA cylinder and helical ribbon structures}

As mentioned in the previous section, surfactants (like phospholipids in water) tend to aggregate in solution forming bilayer membrane structures. Surfactant molecules can self-assemble to form not only flat bilayers, but also twisted or helical ribbons, tubules and cylinders. [51] The morphology and internal structure of the assemblies are defined by molecular architecture and directional associative interactions (physical bonds) between molecular centers and functional groups (ligands), [52] but the presence of chiral centers is important as well. [52-56]. The role of chirality (molecular handedness) was elucidated in many theoretical studies of amphiphilic self-assemblies in solution. [51] In particular, formation of tubular 
and ribbon-like structures had been explained using a phenomenological approach based on the continuous elasticity theory of chiral anisotropic membranes. [55-60] Within this approach the molecular chirality is expressed in terms of the mirrorsymmetry breaking contribution to the elastic bending energy of a tilted bilayer membrane: $[58,59,56]$

$$
f_{\chi}=\chi \operatorname{Tr}(\underline{\underline{C}} \cdot \underline{\underline{S}} \cdot \underline{\underline{\epsilon}})
$$

where $f_{\chi}$ is the chiral energy per unit area, $\chi$ is the degree of chirality, $\underline{\underline{S}}=$ $S(2 \underline{m m}-\underline{\underline{\delta}}), S$ is the 2-dimensional (2D) nematic order parameter (related to the tilt angle), $\underline{m}$ is unit $2 \mathrm{D}$ vector defining the tilt direction, $\underline{\underline{\delta}}$ is the unit $2 \times 2$ matrix, $\underline{\underline{\epsilon}}$ is the antisymmetric $2 \times 2$ matrix symbol, and $\underline{\underline{C}}$ is the membrane curvature matrix. This term should be added to the standard Helfrich bending energy augmented with the anisotropic $K^{\prime}$-term:

$$
f_{0}=\frac{K}{2}(\operatorname{Tr} \underline{\underline{C}})^{2}-K_{G} \operatorname{det} \underline{\underline{C}}+\frac{K^{\prime}}{2} \operatorname{Tr}\left(\underline{\underline{C}}^{2} \cdot \underline{\underline{S}}\right)
$$

where $K$ is the membrane bending modulus, $K^{\prime}$ reflects the bending anisotropy, and $K_{G}$ is the Gaussian modulus. The energy $f_{\chi}$ favors spontaneous cylindrical curvature of the initially flat bilayer in the direction forming an angle $\phi$ with respect to the nematic director $\underline{m}$ ( $\phi$ is close to $\pm 45^{\circ}$ for low anisotropy of membrane elasticity, $K^{\prime} S$ ) [58,59,51]. As a result, the bilayer tends to form a tube (hollow cylinder) with radius $r \propto K /(\chi S)$. The predicted cylinder radius thus diverges in the nonchiral case $\chi=0$; it also diverges at $S=0$, that is, with no molecular tilt. The theory thus implies that the actually observed microtube morphologies must be chiral. This conclusion was verified in circular dichroism studies on lipid systems. [51] On the other hand, highly curved SA morphologies (like hollow nanotubes or helical ribbons) have been observed also with totally achiral molecular blocks. [91] A similar behavior was found in experiments on diacetylenic lipid tubules formed in a mixture of $\mathrm{D}$ and $\mathrm{L}$ molecular enantiomers: the tubule radius did not depend on the enantiomeric composition. [62] These observations indicate that a different mechanism unrelated to the intrinsic molecular chirality must be responsible for highly curved structures at least in some surfactant systems. Such alternative mechanisms leading to spontaneously chiral SA morphologies are considered in the next section 5. Right below we turn to twisted bilayer ribbons and related structures which bear some similarity to those discussed in section 4.3).

\subsection{Twisted membrane structures of chiral surfactants}

The studies described above $[58,59,51]$ largely assumed that the membrane curvature is cylindrical, $\operatorname{det} \underline{\underline{C}}=0$. This assumption is supported by numerous experimental data revealing formation of nano- and micro-tubules or helical ribbons in solutions of amphiphilic molecules. [51,63,64,43] However, other morphologies involving Gaussian (saddle-like) curvature, $\operatorname{det} \underline{\underline{C}} \neq 0$, can as well be competitive. The most important example of that sort of structure is provided by twisted ribbons, Fig. 9. $[54,55,65,63,66]$ The role of Gaussian curvature can be clarified based on the elastic bending energy $f_{\mathrm{el}}=f_{0}+f_{\chi}$ given in eqs. (31), (32). Let us use the Cartesian reference frame with axis 1 along the $2 \mathrm{D}$ nematic director $\underline{m}$ and axis 2 
perpendicular to it. The elastic energy then reads $f_{\mathrm{el}}=K_{G} C_{12}^{2}+2 \chi S C_{12}+P_{2}\left(C_{11}, C_{22}\right)$, where $C_{11}, C_{22}, C_{12}$ are the independent components of the curvature matrix and $P_{2}$ is a quadratic form. The stability of a flat bilayer at $\chi=0$ dictates that $P_{2}$ must be positively defined and $K_{G}>0$, hence the minimum of $f_{\mathrm{el}}$ at $\chi \neq 0$ must correspond to

$$
C_{11}=C_{22}=0 \text { and } C_{12}=-\chi S / K_{G}
$$

that is to a purely saddle-like surface with zero mean curvature $\left(C_{11}+C_{22}=0\right)$ and the angle $\phi=45^{\circ}$ between $\underline{m}$ and the principal curvature axes. The conditions (33) can be nearly satisfied for narrow twisted ribbons with pitch $P$ and width $W$, $P \gg W$ (cf. Fig. 9). [55,56] Such twisted ribbon structures have been observed in aqueous solutions of dicationic gemini surfactants with chiral counterions [55]. Both D- and L- counterions and their mixtures have been shown to generate uniform ribbons whose pitch and width change continuously as a function of the enantiomeric excess (ee) of the counterions. [55] They also attempted to qualitatively rationalize the experimental dependencies $P(e e), W(e e)$ using a simple model similar to that described just above (and assuming that $\chi$ is proportional to $e e$ for small ee): It was thus shown [55] that twisted ribbons are selected thermodynamically (as compared to flat bilayers or flat ribbons) if the chiral energy parameter $\chi S$ is high enough, $\chi S / \gamma \gtrsim 1$, where $\gamma$ is the energy penalty per unit length of the ribbon edge. However, a more detailed theoretical analysis [56] has highlighted important discrepancies between the data [55] and their model. In particular, the data point to $P / W \propto 1 / e e$, while the model predicts that equilibrium $P / W$ increases with ee. $[56]^{2}$

The theory was further generalized to account for multi-lamellar membranes [56] stabilized by face-to-face attraction between the stacked bilayers (in the case of charged surfactants the stacking is facilitated by counterions located between consecutive bilayers). The optimum number of lamellar layers $N^{*}$ was shown to be finite and to decrease at larger $\chi S$. However, the predicted pitch-to-width ratio $P / W$ still increases with $\chi S$ in the same manner as for single bilayers.

All the models considered above in this section assumed that the bilayers are liquid-like ( $L_{\alpha}$ phase). However, there is a lot of evidence that surfactant bilayers are characterized by pseudo-crystalline $2 \mathrm{D}$ positional order $\left(L_{\beta}\right.$ gel-phase). [67-69,63,66] In particular, such crystalline bilayer organization was revealed, both by WAXS and FTIR, in all gemini surfactant systems producing SA ribbons. [63,66] It was argued, however, that the positional order is limited to 2 dimensions and does not lead to a 3 -dimensional order due to rather weak lateral coupling between neighboring bilayers. $[56,66]$ The pseudo-crystalline bilayer structure generally favors morphologies with cylindrical curvature (like tubules), since layers with saddle-like curvature involve 2D shear deformations with rather high

${ }^{2}$ The dependence $P / W \propto 1 /$ ee in the regime $\gamma \gg \chi S$ can be theoretically justified assuming that the ribbon width $W$ (or its length and area) is kept constant for some reason $[55,60,56]$. However, it is not clear why the width of a liquid-like ribbon should adopt a non-equilibrium finite value (which, besides, is independent of the degree of twisting!) given that, according to the theory, a flat layer morphology with $W \rightarrow \infty$ must be selected thermodynamically in the considered regime. 
energy penalty proportional to the shear elastic modulus $G$. However, low values of $G$ are expected due to a weak degree of 2D crystalline order (in particular, close to $L_{\beta}-L_{\alpha}$ transition) associated with a high density of defects, [57,70-72] hence the twisted ribbon morphology can be stabilized also with bilayers in the $L_{\beta}$ phase (note that this phase also implies a high bending modulus $K$, hence a high energy penalty for cylindrical bending). [56] In addition, low ribbon edge tension $\gamma$ (which can be expected in charged surfactant systems due to electrostatic repulsion between the edges) also favors the twisted ribbon morphology. The stability of twisted ribbons with pseudo-crystalline bilayers was addressed in ref. [56]. In particular, it was found that the ratio of the ribbon pitch to width, $P / W$, decreases as the degree of chirality $(e e)$ is increased (this dependence is more pronounced for multi-lamellar ribbons) in qualitative agreement with experimental data [55]. These results suggest that ribbon structures observed in gemini surfactants $[63,66]$ are likely to involve multi-lamellar membranes in the gel phase.

To resume, the continuous membrane elasticity theory proved to be successful in explaining the salient morphological features of SA layers for a range of surfactant systems (including phospholipids in water). The theory predicts that chiral bilayers with molecular tilt have an intrinsic tendency for bending. [58] This tendency leads to different competing layer morphologies with cylindrical or saddle-like curvature. The common view is that cylindrical structures are expected for surfactant bilayers in the gel phase [73], while twisted ribbons are formed in the fluid phase. It was argued, however, [51] that membrane morphologies with cylindrical curvature can be thermodynamically stable for bilayers in a fluid phase in spite of a somewhat controversial experimental evidence. Generally, we support this view on the theoretical grounds (if the fluid membrane can be still anisotropic): the fluid-phase cylindrical structures can be certainly favored if $K_{G} / K$ is not low, and/or if the ribbon edge energy $\gamma$ is high enough since the edge length of long cylindrical molecular tubes is negligible. Noteworthily, the ultimate stability of cylindrical bilayers does not exclude their temporal coexistence with metastable twisted ribbons. [55] By comparison, one of the main conclusions of ref. [56] is different: the twisted ribbon morphology can be thermodynamically favored also with a gel $\left(L_{\beta}\right)$ bilayer phase if the edge energy $\gamma$ and the bilayer shear modulus $G$ are low enough or if the bending modulus $K$ and the degree of chirality are high.

\subsection{Fibrillar structures in peptide solutions}

Let us discuss frustrated structures of designed peptides in aqueous solutions where each peptide unit (aminoacid residue) can be considered as a SIG capable of forming two H-bonds. [79] As was demonstrated in the pioneering study [74], the rationally designed oligo-peptides under appropriate conditions spontaneously self-assemble in filament structures which can serve as a basis of a wealth of hierarchically organized materials. In particular, a family of short designed peptides of 5-24 aminoacid units show a strong tendency towards formation of regular $\beta$-sheets structures, Fig. 10a. The resultant long $\beta$-sheet tapes of identical peptide molecules in aqueous solution [75] are stabilized by multiple inter-molecular hydrogen bonds and other side-group recognition interactions between neighboring peptides. The peptide molecules adopt stretched rod-like conformations in this 
tape structure, Fig. 10b. Concomitantly, the ends of side-groups paving the tape surfaces attract each other leading to the possibility of face-to-face stacked $\beta$-sheets, Fig. $10 \mathrm{c}$ (with energy gain $\sigma$ per unit area). If the tapes were perfectly straight and flat, their stacking would result in infinite 2D aggregates of parallel tapes, Fig. 10d. Such simple aggregation can be reproduced with a toy-model, where peptide molecules are represented as cuboids which laterally attract each other (with moderate recognition of similar rectangular faces), Fig. 10e. However, if the shape of building blocks deviates from a perfect cuboid (for example, is weakly twisted, Fig. 10f), their packing in infinite 2D or 3-dimensional (3D) aggregates may become problematic. Instead, long fibrillar (string-like) structures may be favored for screw-like blocks, Fig. 10h,i. Indeed, oligo-peptide fibrils of finite and well-defined thickness (corresponding to a few tapes stacked together) have been observed in different studies. [74,76,77] This phenomenon was attributed $[75,78,79]$ to the fact that natural aminoacids are chiral, hence the primary $\beta$-sheet tapes must be twisted, Fig. $10 \mathrm{~g}$. The mechanism of their frustrated self-assembly have been elucidated in refs. $[75,78,79]$ as outlined below.

The equilibrium state of an isolated $\beta$-sheet tape implies a regular rotation of the local tangential plane (defined by the normal vector $\underline{n}$, cf. Fig. $10 \mathrm{~b}$ ) along the tape axis $(s)$; the corresponding twist period is $h_{0}$. The face-to-face stacking of several tapes along the direction $\underline{n}=\underline{n}(s)$ is frustrated due to the twist (rotation of $\underline{n}$ as a function of $s$ ): the outer tapes in the stack (cf. Fig. 10h) have to entwine round the axis of the screw-like structure. Thus, the local twist strain of peripheral tapes decreases (becoming less optimal) and their axes become necessarily bent, see Fig. 10i. These elastic distortions and the corresponding energy penalties (determined by the respective tape bend and twist rigidities, $k_{\text {bend }}$ and $k_{\text {twist }}$ ) aggravate for thicker stacks. As a result, the elastic energy cost for stacking overwhelms the face-to-face attraction energy gain for thick enough stacks. This mechanism provides thermodynamic selection of fibrils with well-defined thickness corresponding to some optimal number $p$ of tapes per fibril.

The results of the theoretical analysis [75] of such fibril stabilization by twist are illustrated in the diagram of regimes, Fig. 11, for the experimentally most relevant case of weakly twisted tapes, $\widetilde{a} \equiv a / h_{0} \ll 1$ (cf. Fig. $10 \mathrm{~g}, \mathrm{~h}$ ). The following dimensionless parameters are used:

$$
\tilde{k} \equiv k_{\text {bend }} / k_{\text {twist }}, \quad \tilde{\sigma} \equiv \sigma a b h_{0} / k_{\text {twist }}
$$

where $a b$ is the cross-section of the tape, cf. Fig. 10d,h). If the face-to-face attraction is weak $\left(\tilde{\sigma}<\tilde{\sigma}_{*}\right)$, the tapes do not aggregate and single tapes are stable. As attraction increases, a first order phase transition from single tapes to stacks (fibrils of $p_{*}$ tapes) takes place at $\tilde{\sigma}=\tilde{\sigma}_{*}$ :

$$
\widetilde{\sigma}_{*} \sim\left\{\begin{array}{cc}
\tilde{a}^{5}, & \text { if } \tilde{k} \lesssim \widetilde{a}^{2} \quad \text { (regime LB); } \\
\widetilde{k} \widetilde{a}^{3}, & \text { if } \widetilde{a}^{2} \lesssim \widetilde{k} \lesssim \widetilde{a}^{-2} \quad \text { (regime MB); } \\
\widetilde{a}, & \text { if } \tilde{\vec{k}} \gtrsim \widetilde{a}^{-2} \quad \text { (regime HB) }
\end{array}\right.
$$

(see ref. [75] for the numerical prefactors omitted here and below). The transition to the simplest fibril with $p_{*}=2$ (double tape or ribbon) occurs in the regime of low or moderate bending modulus $\left(\widetilde{k} \lesssim \widetilde{a}^{-2}\right)$, while for high bending modulus (regime HB) multiple stacks with $p_{*} \simeq \widetilde{k} \widetilde{a}^{2} \gg 1$ are formed directly. A further increase of 
the face-to-face attraction (in the range $\tilde{\boldsymbol{\sigma}}_{*}<\tilde{\boldsymbol{\sigma}} \lesssim 1$ ) leads to secondary transitions with formation of thicker fibrils whose reduced thickness grows approximately as $p \tilde{a} \propto \tilde{\sigma}^{1 / 5}$ (in LB), $p \tilde{a} \propto(\tilde{\sigma} / \tilde{k})^{1 / 3}$ (in MB) or $p \tilde{a} \propto \tilde{k} \tilde{\sigma}^{3}$ (in HB). In the regimes LB and MB the twist period $h$ of the optimal stacks stays approximately equal to the original twist period $h_{0}$. However, in the regime HB the fibrils are much less twisted than the isolated tapes: $h / h_{0} \simeq \widetilde{k} \tilde{\sigma}^{2} \gg 1$. In all three regimes, LB, MB and HB, the fibrils stay much thinner than the twist period: $p a / h \ll 1$. As the attraction approaches the second critical line $\tilde{\sigma}=\tilde{\sigma}_{* *}\left(\tilde{\sigma}_{* *} \simeq 2 \pi^{2}\right)$ from below $\left(\tilde{\sigma}_{* *} / 2<\tilde{\sigma}<\tilde{\sigma}_{* *}\right)$, the system enters another scaling regime of thick fibrils (regime WF) where the optimal fibrillar stacks are weakly twisted:

$$
\frac{h}{h_{0}} \simeq \frac{1+\tilde{k}}{\widetilde{\sigma}_{* *}-\tilde{\sigma}} \gtrsim 1, \quad p \tilde{a} \simeq \frac{1+\tilde{k}}{\left(\tilde{\sigma}_{* *}-\tilde{\sigma}\right)^{2}}
$$

(note that $p a / h \equiv p \tilde{a} h_{0} / h \sim\left(\tilde{\sigma}_{* *}-\tilde{\sigma}\right)^{-1} \gtrsim 1$ in this regime). At the line $\tilde{\boldsymbol{\sigma}}=\tilde{\boldsymbol{\sigma}}_{* *}$ the fibrils undergo a second-order phase transition resulting in formation of flat infinite 2D sheets, $p \rightarrow \infty, h \rightarrow \infty$ (regime 2D).

The designed peptides also show an interesting behavior as concentration is changed. A representative set of filament structures (Fig. 12) was observed and theoretically elucidated $[78,79]$ for realistic model peptides requiring an activation in order to enter a $\beta$-sheet tape structure (with activation energy $E_{\text {tr }}$ ). The peptide unimers form asymmetric SA tapes with two distinct faces, Fig. 12ac. One surface of a tape (red in Fig. 12c) is hydrophobic, hence a double-tape aggregation (ribbon formation, cf. Fig. 12d) is favored above some very low critical aggregation concentration (CAC) defined by the 'red' face-to-face attraction energy per rod-like peptide, $E_{\text {red. }}$. Another (grey) face of a tape is less insoluble, but the corresponding 'grey' face-to-face attraction (with energy $E_{\text {grey }}$ per rod) is still strong enough to cause face-to-face stacking of double-tapes at higher concentrations (cf. Fig. 12e). Such fibrillization takes place above the second CAC (related to $\left.E_{\text {grey }}, E_{\text {grey }}<E_{\text {red }}\right)$. [78,79]. The above scenario accounts for the basic features of oligo-peptide $\beta$-sheet self-assembly: On the one hand, before entering the tape the peptides have to abandon their solvent-friendly coil or $\alpha$-helix state and transform to a $\beta$-tape competent extended (rod-like) state. On the other hand, the peptides are necessarily anti-parallel in the $\beta$-sheet structure (to correctly saturate all the $\mathrm{H}$ bonds), hence the tape faces are generally distinct. The evolution of SA structures with the unimer peptide concentration is predicted to be similar to that shown in the sequence of Fig. 12c-f if

$$
E_{\text {tr }}>k_{B} T \sim E_{\text {red }}>E_{\text {grey }}
$$

As concentration increases the more developed string-like structures emerge once the already formed simpler filaments grow long enough to allow for the new structure formation. The family of model $\beta$-sheet oligopeptides synthesized in Leeds $[76,77,80]$ consistently demonstrate the predicted behavior $[78,79,81,82]$.

The fibril structure and thickness are thus defined by a frustration effect stemming from a contradiction between the natural twisted conformation of individual SA tapes and their tendency towards face-to-face stacking implying helical deformations of the tape axes. The developed theoretical model has the potential to guide the design of new functional peptides capable of forming SA filaments 
of a desired type at prescribed conditions by tuning the peptide length, the positions of charged side groups and their nature (thereby changing the relevant attraction energies, cf. eq. (37), in a predictable way). Such development is important since the SA peptide systems proved to combine a range of useful properties, such as biological functionality, high mechanical strength, lability, responsiveness to external chemical and physical triggers (solvent polarity, temperature, $\mathrm{pH}$, ionic strength) and ability to aggregate at extremely low peptide concentrations. $[74,76,81,82]$ The one-dimensional peptide self-assembly thus opens a promising route for production of artificial nano-structured functional materials for biomedical and other applications offering numerous benefits of being cheap, biocompatible and biodegradable. $[77,79,83,80]$ It is also worth mentioning that twisted $\beta$-sheet fibrils are similar to extra-cellular proteinaceous fibres encountered in various amyloid diseases, including Alzheimer's, prion and Parkinson's. [84,85]

\subsection{Chiral recognition effects in DNA compaction}

In the previous parts $4.1,4.2$ we considered the effects of molecular chirality on the morphology of highly-curved SA layer structures. Within the continuummechanical phenomenological approach described above the effective chirality of a mixture of enantiomers is introduced by means of a single parameter $\chi$ disregarding the exact molecular packing structure. Such approach is justified if the aggregate is much thicker than the molecular size and if the chiral asymmetry of molecular enantiomers just weakly affects their local packing structure. The latter condition is not met in many systems where chiral molecular stacking and phase separation of enantiomers are possible (cf. sections 4.3, 5.2).

An example of a system where local molecular recognition is important for the global structure is considered below. Here, instead of many small molecules we consider a long double-strand DNA polymer with many amphiphilic base pairs. The DNA units get negatively charged in water, so a long DNA macromolecule typically adopts a loose random-coil conformation (Fig. 13a) due to strong electrostatic repulsions of its segments. These repulsions can be suppressed by adding multivalent cations neutralizing the DNA charge and leading to formation of compact DNA globules (Fig. 13b). The volume occupied by the DNA molecule dramatically decreases during the condensation transition. [86] Compact DNA states are very important in vivo defining how the genetic information is being stored and manipulated. DNA globules are typically torroidal with locally parallel alignment of neighboring chain fragments due to their high stiffness. It is therefore an interaction between parallel DNA segments that is crucial for the DNA compaction.

A remarkable study [87,88] of a double-strand DNA compaction transition (the number of DNA base-pairs is $N_{\mathrm{bp}} \approx 160000$ ) induced by diammonium cations (Fig. 13c,d) revealed an unusual chiral discrimination effect (cf. Fig. 13e): the chiral SS2-form of dications was very efficient in promoting the DNA condensation (at dication concentration $c \sim 10^{-3} \mathrm{M}$ ), while its mirror image RR2 (the isomer of the same molecule with the opposite sense of chirality) does not induce compaction at all for $c<0.1 \mathrm{M}(\mathrm{M}=\mathrm{mol} / \mathrm{L})$. Moreover, the DNA compaction ability of SS2 isomers was strongly suppressed in the presence of RR2 enantiomers. [87]

These striking chirality effects have been explained by the chiral recognition theory [89]. DNA compaction is normally impossible without its charge neutral- 
ization required to suppress strong electrostatic repulsion of its segments. The terminal groups of ammonium dications [87,88] can form 2-3 hydrogen bonds with an oxygen $\left(\mathrm{O}^{-}\right)$of a DNA phosphate group. Moreover, a dication can form bonds with 2 such groups since its length $(\approx 1 \mathrm{~nm})$ matches well the distance between the neighboring phosphate groups across the DNA minor groove (Fig. 13f). Thus, the DNA binding potential of the dications must be very high as was indeed confirmed by the all-atomic simulations [89] showing that all phosphate groups get neutralized by dications in the concentration range studied experimentally $\left(10^{-6}-10^{-1} \mathrm{M}\right)$. This effect leads to a dramatic decrease of the DNA net charge by a factor of $\sim 10^{3}$ or higher.

A more detailed analysis [89] showed that the residual electric field of the DNA is very weak and short-ranged, so it can be totally neglected at the distance $h \gtrsim 1 \mathrm{~nm}$. We therefore have to conclude that electrostatic interaction between DNA segments is negligible. The natural question is: what is then the driving force for DNA condensation? It is likely to be a specific interaction of non-electrostatic origin. The central idea [89] is that dications can form bridges between neighboring DNA fragments thus providing their attraction (Fig. 13g). Indeed, it was found that the binding energy of a bridge at the optimum separation $h^{*} \approx 1.1 \mathrm{~nm}$ is higher than that for the same dication adsorbed on a single DNA molecule (cf. Fig. 13g). Further, it was revealed [89] that the binding constants $K_{S}$ and $K_{R}$ for adsorption, respectively, of SS2 and RR2 dications on the chiral DNA surface are significantly different, with $K_{R}>K_{S}$. This stereoselectivity is provided by the chirality-sensitive interaction of dioxolane ring (cf. Fig. 13c,d), with the minor groove (cf. Fig. 13f). On the other hand, the dication bridging activities are nearly the same (with the binding constant $K_{b}$ ). Thus, $K_{b}>K_{R}>K_{S}$ (cf. inset of Fig. 13e).

The chiral discrimination effects mentioned above can be explained on this basis as follows: Suppose chiral dications SS2+ are added to the DNA solution. The dications must strongly tend to form bridges between neighboring DNA fragments (with separation $h \approx h^{*}$, cf. Fig. $13 \mathrm{~g}$ ) if their concentration $c \gg c_{b}=1 / K_{b}$, where $K_{b}$ is defined by the bridging energy $E_{b}: \quad K_{b} \propto \exp \left(E_{b} / k_{B} T\right)$. Therefore, the threshold concentration for bridging is exponentially low for high $E_{b}$. Moreover, nearly all possible bridges will be formed if the bridging activity $K_{b}$ is much higher than the dication adsorption ('clinch') constant $K_{S} \propto \exp \left(E_{S} / k_{B} T\right), K_{b} \gg K_{S}$ (the maximum number of bridges is $p N_{\mathrm{bp}}$ where $p=0.1$ corresponds to one bridge per helical turn involving 10 base-pairs) [89]. As a result the DNA compaction can be induced by SS2+ cations at a low concentration comparable with $c_{b}$. On the other hand, the RR2+ enantiomer cations have higher ability to bind on a single DNA fragment $\left(K_{R}>K_{S}\right)$ which competes with their bridging activity. This competition yields a low fraction of bridges saturated for $c \gg c_{b}$ at a level much smaller than $p$, which is not enough for DNA compaction. Finally, in a mixture of SS2 and RR2 cations, the adsorbed RR2 isomers (bound to single DNA) compete for phosphate groups with SS2 bridges, so the number of bridges decreases as well, leading to significantly higher dication concentrations required for compaction.

It was found [89] that the theory can quantitatively reproduce the experimental behavior [87] with $K_{b} / K_{S}=20$ and $K_{b} / K_{R}=4.55$ (this means that the effective bridging energy is higher than the SS2-adsorption energy by $\approx 3 k_{B} T$, while the analogous energy difference for RR2 enantiomers is about $1.5 k_{B} T$ ). The comparison 
of experimental and theoretical results is shown in Fig. 13e. The figure clearly demonstrates a highly antagonistic behavior of the cationic enantiomers reflected in low compaction activities of their mixtures. Recalling that DNA condensation and decompaction are among the most crucial biological processes, it is possible to assume [89] that the elucidated chiral selectivity effect may serve as a part of a general molecular mechanism leading to biological homochirality.

\section{Spontaneous chirality}

In the previous section we considered helical and twisted membranes and other structures involving chiral amphiphilic molecules. However, mirror-symmetry-breaking SA morphologies can be also formed in systems of totally achiral molecules (both surfactant and solvent). In such systems the chirality of molecular aggregates is driven spontaneously by cooperative effects. [90-97] Below we survey some phenomenological and other approaches to the problem.

\subsection{Landau-type theories of tape and helical structure stabilization by spontaneous chirality}

A widely recognized theory of spontaneous chiral symmetry breaking in tilted lipid bilayers was proposed rather long ago [98]. It is based on the phenomenological approach outlined in section 4.1 augmented by a physically reasonable assumption of weak interdigitation between the constituent lipid monolayers. They introduced two weakly coupled nematic directors, unit vectors $\underline{m}_{1}$ and $\underline{m}_{2}$, defining the direction of molecular tilt in each monolayer (cf. Fig. $4 c$ ). Interactions between the sublayers favor antiparallel alignment of $\underline{m}_{1}$ and $\underline{m}_{2}$ with $\alpha=0$ (cf. Fig. 14), where $\pi-\alpha$ is the angle between the two directors. The energy penalty for nonparallel $\underline{m}_{1}$ and $-\underline{m}_{2}$ is given by the aligning potential [98] $f_{\text {ap }}=(\Gamma / 2) \sin ^{2} \alpha$, $\alpha \leq \pi / 2$ (cf. Fig. 14c). The faces of each monolayer are different: one face is solvophilic (heads), another is solvophobic (tails), so a separate monolayer must have an intrinsic tendency to bend which can be described by a linear contribution to its elastic bending energy: $E_{\mathrm{lb}}(\underline{m})=$ const $\operatorname{Tr} \underline{\underline{C}}-\beta \operatorname{Tr}(\underline{\underline{C}} \cdot \underline{m m})$, where the second term accounts for the anisotropy of the tilted sublayer and $\underline{m}=\underline{m}_{1}$ for the first monolayer, while $\underline{m}$ must be replaced with $\underline{m}_{2}$ and $\underline{\underline{C}}$ with $-\underline{\underline{C}}$ for the second monolayer. With $\underline{m}_{2}=-\underline{m}_{1}$ the two linear contributions (coming from both monolayers) completely compensate each other. In the general case the total linear energy is

$$
f_{\chi} \equiv E_{\mathrm{lb}}\left(\underline{m}_{1}\right)-E_{\mathrm{lb}}\left(\underline{m}_{2}\right)=\beta \sin \alpha\left(C_{1}-C_{2}\right) \sin (2 \phi),
$$

where $C_{1}$ and $C_{2}$ are the principal curvatures of the bilayer and $\phi$ is the angle between the principal axis 1 and the median of vectors $\underline{m}_{1}$ and $-\underline{m}_{2}$ (cf. Fig. 14d). (Note that eq. (38) is equivalent to eq. (31) with $\chi S=\beta \sin \alpha$.) Minimizing the total elastic energy, $f=f_{0}+f_{\chi}+f_{\text {ap }}$ ( $f_{0}$ is the standard Helfrich bending energy defined in eq. (32)), with respect to curvatures $C_{1}, C_{2}$ one finds that the flat achiral state is stable if $0<K_{G}<2 K$ and $\beta$ is small (here we neglect the bending anisotropy, setting $K^{\prime}=0$ for simplicity), but otherwise, for $\beta^{2}>\Gamma K_{G} / 2$, the minimum of the free energy per unit area corresponds to a curved and chiral 
state $\left(C_{2}=-C_{1}= \pm \beta / K_{G}, \alpha=\pi / 2\right)$. It is important, however, that the above conditions cannot be satisfied globally if the layer is kept topologically equivalent to a plane surface. In the latter case a highly curved and chiral bilayer ground state is predicted for $\beta^{2}>\Gamma K$ (with $\left|C_{2}-C_{1}\right|=\beta / K, \alpha= \pm \pi / 2$ and $\phi= \pm \pi / 4$ ). [98] A one-dimensionally modulated ripple phase was proposed as an example of such distorted ground state [98].

The mechanism of spontaneous parity breaking outlined above can be applied to a liquid-like bilayer with 2D nematic order and weak coupling between the sublayer directors (small $\Gamma$ ). Noteworthily, this physical mechanism implies simultaneous emergence of spontaneous curvature and spontaneous chirality of the membrane. Nevertheless, there are many systems where the spontaneous chirality is not related to the curvature which, in turn, is not defined by the chirality, but rather both effects (curvature and chirality) are dictated by the molecular packing structure. Some examples of such systems are discussed below (and, in more detail, in sections 5.2 and 5.3).

Another theoretical approach to chiral symmetry breaking in anisotropic amphiphilic layers involves a coupling between molecular or structural chirality with modulations of the nematic director. [99] This physical mechanism invokes the Ginzburg-Landau-type free energy term $-\lambda \chi \nabla \times \underline{m}$, where $\chi=\chi(\underline{r})$ is the chiral order parameter (the degree of chirality reflecting a locally chiral molecular packing or a local excess of one enantiomer in a globally racemic mixture) and $\lambda$ is the coupling constant. For high enough $\lambda$ the initially achiral system spontaneously develops a chiral pattern (e.g. stripes) with modulations of both $\chi$ and $\underline{m}$. While this concept and related theories (involving the $\lambda$-term) [99-101] can explain certain important features of lipid bilayer mesophases (spontaneous chiralization, modulations of bilayer tubules with helical patterns of stripes and curvature ripples, helical ribbon stability), their general applicability can be questioned. One problem is that the stability of the predicted modulated states crucially depends on the energy of sharp defect lines separating the stripes [51] together with the ribbon edge energy, which cannot be treated within the same (Ginzburg-Landautype) approach as they involve high membrane curvature and rapid variations of 2D nematic director field (and/or of the packing structure) on the molecular length-scale. Secondly, the models considered above assume fluid $\left(L_{\alpha}\right)$ membrane phase, while all the cylindrical (tubular) morphologies of lipid bilayers and related patterns are mostly observed in the more ordered tilted gel phase ( $L_{\beta^{\prime}}$ phase) below the main transition temperature. [102-104] We also note in passing that the striped/ripple patterns mentioned above bear some similarity to ripple phases of lipid bilayers [103,104].

The spontaneous parity breaking generally involves a subtle interplay of local chirality, curvature, nematic fields and the local positional order. These and other factors together point to the molecular conformational lability and a frustrated packing structure as the main driving force for spontaneous chiralization. For example, it was shown [65] that the membrane-forming gemini cationic amphiphiles considered in section 4.2 generically exist as a mixture of chiral conformers and that tartate counterions specifically interact with these conformationally labile cations thus inducing a global chirality of the membrane. Therefore, some tendency for the parity breaking may be coded already in stereostructure of achiral gemini molecules and their bilayer packing mode. 
Another example concerns diacetylenic lipids which show a high propensity to form tubules even in a globally achiral racemic mixture [105,62]. It was noted [62] that a kink in the middle of the acyl chain (due to the diacetylene group) might favor non-parallel twisted packing of neighboring chains leading to a chiral symmetry breaking which could be biased by the majority handedness. While such an argument could possibly explain why the radius of tubules formed in mixtures of $\mathrm{D}$ and $\mathrm{L}$ lipids was found to be independent of ee, [62], the CD measurements [62,51] rather indicate that a racemic mixture of the lipid enantiomers strongly phase separates to form left- and right-handed tubules [105], probably for similar packing reasons.

Chiral morphologies (including helical ribbons) of achiral amphiphilic molecules (azobenzene-containing fatty acids) have been reported in a recent paper [90] suggesting that chiral stacking of molecules in bilayers is due to the presence of rigid azobenzene groups working as asymmetric elements (kinks). Self-assembly of helical nano-ribbons was also observed in aqueous solutions of terpyridine-based molecules with no chiral center [94]. The spontaneous morphological chirality was attributed to 2 possible twist conformations of the wing pyridine groups (enantiomeric molecular isomers) leading to a facilitated orderly stacking of similar chiral isomers [94] (cooperative isomer selection) and spontaneous handedness of the whole stacked structure. Interestingly, this mechanism seems to work in spite of the dynamical (rather than chemically quenched) nature of chiral isomers.

A similar behavior was observed in co-assembly of 2 distinct types of achiral organic molecules [95]: These amphiphilic entities first form cyclic hexamer supermolecules existing in 2 chiral enantiomeric (propeller-like) configurations; the chiral supermolecules then self-assemble in long mesoscopic right- or left-handed supercoil structures. Noteworthily, several other studies [106-108] also underlined the importance of 'propeller-like' conformations of achiral molecular unimers for the chiral symmetry breaking.

In the next subsections we consider SA structures of achiral amphiphilic molecules with SIGs whose packing arrangements and chiral symmetry breaking mechanisms are described in detail at a submolecular level.

\subsection{BHPB structures}

This section is devoted to SA membrane structures of organogelator molecules involving strongly interacting groups (SIGs) of two types [109-111]. The generic chemical structure of these aromatic diamide-ester molecules (called BHPB-n) is shown in Fig. 15a,b: each molecule consists of alkyl fragments (soluble in organic solvents like cyclohexane) and insoluble SIGs, namely, one aromatic-ester ring ('II') and two amide groups ('IV'). The similar groups tend to form distinct layer structures stabilized by favorable interactions and reversible bonds between the SIGs. Aromatic-ester groups associate due to $\pi-\pi$, dipole-dipole and quadruple interactions, while the amide layer recognition structure is supported by H-bonds and dipole-dipole attractions.

The BHPB molecules can self-assemble in both monolayer and bilayer membranes, Fig. 15c,d. The major difference between monolayer and bilayer structures is related to the number of amide sublayers demanding that the $2 \mathrm{D}$ number density of rings must be twice lower than that of amide groups in monolayers (with 
1 amide sublayer), while both densities must be similar in bilayers (with 2 amide sublayers). [112] These geometrical conditions define the most favorable 2-dimensional (2D) arrangements of aromatic rings and amide groups in the monolayer and bilayer membrane structures, as obtained by all-atomic computer simulations [112] and shown in Figs. 15e-h. It was found [112] that bilayer structures are more favorable thermodynamically for $n \leq 7$ (where $n$ is the number of $\mathrm{CH}_{2}$ units in the ester-tail 'I', cf. Fig. 15a,b). On the other hand, the bilayer morphology is not allowed for sterical reasons if $n \geq 8$ (in this case ester tails cannot be accommodated in the alkyl regions 'III+I' whose thickness is defined by the alkyl spacer length $n_{s}=5$, cf. Fig. 15d).

Noteworthily, both membrane structures involve several types of frustrations:

(i) Sublayer area mismatch: The packing of alkyl spacers in the monolayer structure demands that the area per spacer is $\geq 17 A^{2}$, hence the area per aromatic ring must exceed $34 A^{2}$, which is greater than the optimum area per ring $S_{\text {ring }} \approx 30.4 \AA^{2}$ dictated by the most favorable internal structure of the aromatic sublayer (cf. Fig. 15e). Therefore, the ring sublayer has to be dilated. As argued in ref. [112] the eventual structure of the aromatic sublayer must involve somewhat separated $\pi-\pi$ stacked columns of the ester-rings (Fig. 16a). A similar dilation effect applies also to the bilayer structure, where there is a mismatch between the optimal area $S_{\text {ring }}$ and the area per amide $S_{\mathrm{am}} \approx 33.5 A^{2}$ in the optimal amide sublayer (cf. Fig. 15g,h); [112] (note that a compression of the amide sublayer is virtually prohibited energetically).

(ii) Spontaneous chirality: It is important that not only the optimum areas but also the packing structures of the aromatic and amide sublayers are different. The ground-state configuration of an aromatic ring and two alkyl spacers attached to it dictates that the $\mathrm{O}-\mathrm{H}$ dipoles of the corresponding amide groups make an angle of $\sim 45^{\circ}$ with the membrane plane (Fig. 17a). By contrast, the simulated preferred amide sublayer structures [112] imply that all lines of H-bonds (cf. Fig. 15f,h) including the CO-HN dipoles must be parallel to the sublayer. In order to make them parallel, the amide dipoles $(\mathrm{O}-\mathrm{H})$ must be rotated by twisting the spacers 'III' in one or another direction (cf. Fig. 17a-d). For packing reasons the rotation sense must be the same for all amide groups in the sublayer. As a result, the lines of H-bonds in the amide sublayer should make an angle $\pm \alpha$ with aromatic rings (with $\alpha \approx 60^{\circ}$ for the globally monolayer structure, cf. Fig. 17e). As BHPB molecules are nonchiral, the two equilibrium structures with both signs of the rotation angle are energetically equivalent. The resultant monolayer structures are therefore necessarily chiral: their broken parity symmetry resolves the cooperative frustration of molecular packing. Such two membrane structures with opposite chirality senses are shown in Fig. 17f. By contrast, the bilayer structures can be either chiral (if the angular rotations from the plane of aromatic rings to amide dipoles are co-directed in both amide sublayers, so that all alkyl spacers are twisted with the same sense) or achiral otherwise (in the latter case the lines of H-bonds in the two amide sublayers are parallel).

(iii) Intrinsic curvature: The dilated state of the aromatic sublayer implies that its surface tension must be positive leading to a tendency for the sublayer shrinkage perpendicular to the columns (its deformation along the aromatic stacks is strongly unfavorable as it would disrupt the $\pi-\pi$ interactions). This effect as such must lead to some bending the whole monolayer membrane towards the 
aromatic sublayer (as the area per ring tends to get smaller than the optimum area $\approx 34.2 A^{2}$ per 2 amides in the amide sublayer) with an intrinsic curvature $C$ (Fig. 18a) giving rise to a cylindrical morphology with well-defined radius $R=1 / C$ and the cylinder axis parallel to aromatic columns (Fig. 18). This picture is valid for relatively short ester tails $(n \lesssim 10)$. In the case of long $n$ the osmotic pressure of the ester-alkyl sublayer can overcome the tension in the aromatic sublayer, hence the curvature $C$ must change sign for $n>n_{i n v}$. The inverse cylindrical morphology (with amide sublayer closer to the cylinder axis) is thus predicted for $n>n_{\text {inv }}$ [112]. A favorable comparison of this prediction with TEM and SANS data [111] points to $n_{\text {inv }} \sim 15$. By contrast, the dilation of a bilayer does not lead, as such, to its intrinsic curvature since the bilayer membrane is symmetric: its faces are equivalent.

(iv) Edge effects: Another type of frustration directly comes from molecular amphiphilicity: SIGs tend to form highly organized dense sublayers, while the soluble alkyl tails (' $\mathrm{I}$ ' and ' $\mathrm{V}$ ' in Fig. 15a,b) prefer an expansion of the accessible volume. Such expansion is possible near the membrane edge, where the tails can diverge laterally (Fig. 16b). This effect provides a negative contribution $\gamma_{t}$ to the edge tension which is roughly proportional to the square of the tail length, $\left|\gamma_{t}\right| \propto n^{2}$. [112] Thus, for long enough $n, n>n^{*}$, the total edge tension $\gamma=\gamma_{t}+\gamma_{\text {SIG }}$ (where $\gamma_{\text {SIG }}$ is the positive energy penalty for missing bonds at the edges of SIG sublayers) must become negative. This effect leads to stabilization of membrane stripes (Fig. 16b) of finite width $D$, with $D$ depending on $n$. [112]

It is important that the SIG contribution to the edge energy $\gamma_{\text {SIG }}$ depends on the edge orientation: it has a pronounced minimum for the edge parallel to the lines of $\mathrm{H}$-bonds in the amide sublayer. In the case of chiral bilayer the edge can not possibly be parallel to $\mathrm{H}$-bond lines of both amide sublayers, hence $\gamma_{\mathrm{SIG}}$ is always high and the stripe formation is never favorable thermodynamically (since $\gamma>0$ ). For the same reason (high edge energy $\gamma$ ) it is expected that formation (nucleation) of chiral bilayers must be prohibited kinetically. [112] By contrast, achiral bilayers (predicted to be stable for $n \leq 7$ ) can form flat stripes like those shown in TEM images, Fig. 6 of ref. [111].

Let us turn to monolayer morphologies predicted for $n \geq 8$. The monolayers involve just one amide sublayer, so they can develop stripes with side edges parallel to the inter-amide H-bond lines. As discussed above the monolayers show intrinsic cylindrical curvature with the axis parallel to aromatic stacks, i.e. perpendicular to the aromatic rings (cf. Fig. 18a). Therefore, the lines of Hbonds must make an angle $\theta \approx 90^{\circ}-\alpha \approx 30^{\circ}$ with the cylinder axis, so these lines turn helical (Fig. 19a). In the regime of ribbons (for $n>n^{*}$ ) their edges must adopt the similar helical shape (Fig. 19b). The resultant helical ribbons are characterized by radius $R=1 / C$ and helical pitch $H_{P}=2 \pi /(C \tan \theta)$, where $C$ is the intrinsic curvature. For geometrical reasons the ribbon width $D$ cannot exceed $H_{P} \sin \theta$. Based on this constraint and the free energy calculation, the following range of the thermodynamically selected BHPB monolayer morphologies have been predicted [112]: uniform hollow cylindrical tubes for $n \lesssim n^{*}$ (Fig. 19d), tubules with helical markings for $n \approx n^{*}$ (Fig. 19c) and helical ribbons for $n \gtrsim n^{*}$ (Fig. 19b). The elucidated chiral morphologies are shown in the phase diagram, Fig. 19e. In addition, a structural transition from bilayer $(n \leq 7)$ to monolayer $(n \geq 8)$ membrane is predicted as $n$ is increased. These predictions [112] are in 
the overall agreement with the experimental data on BHPB solutions $[111,109,110]$ (including quantitative agreement for the hollow tube thickness and the pitch angle $\theta$ with SANS, AFM and TEM data). A discussion clarifying why the tubular morphologies are ubiquitously observed experimentally can be found in ref. [112].

In summary, the study [112] traces down to the atomic level the mechanism of spontaneous parity breaking in self-assembly of achiral amphiphilic molecules containing SIGs giving rise to enantiomeric chiral structures. The physical recognition of similar SIGs define two different highly organized substructures within the same membrane. The geometrical mismatch between the substructures results in packing and orientational frustrations leading to a chiral symmetry breaking in the membrane. The resultant internal spontaneous chirality of the membrane (related to non-parallel anisotropy axes of the sublayers of SIGs) leads to the geometrically chiral helical SA morphologies (hollow nanotubes, tubules, helical ribbons). These results underline the importance of cooperative packing effects and geometrical constraints (including specific directional bonds and steric hindrance effects) for the chiral symmetry breaking in amphiphilic self-assembly of achiral molecules. [90,94-97,106,107]

\subsection{TAA structures}

Let us turn to the recently studied SA nano-objects built with another type of amphiphilic functional units, namely, the triarylamine (TAA) molecules whose chemical structure is shown in Fig. 20. A TAAx molecule consists of the rather rigid arylamine core (central amine and 3 aryl rings), 1 amide group and 2 aliphatic tails, while TATA units have 3 amide groups. These molecules involve 2 types of SIGs: the TAA core and amide groups. The TAA compounds are known as very efficient functional units for semiconductor and optoelectronic devices. [113] However, the ability of TAA molecules for supramolecular self-assembly in dilute chloroform solutions was discovered only recently: It was shown [114] that while all solutions of TAA (with 1 amide group) remain stable in the dark, chloroform solutions of TAAx derivatives shown in Fig. 20a change color from light-yellow to light-green upon exposure to visible light. This color change was attributed to self-assembly revealed, in particular, by AFM images (see Fig. 3 of ref. [114]) showing long bundles of highly packed subfibrils.

The mechanism of this light-induced fibril formation was elucidated both experimentally and by all-atomic modeling [106]. The simulations [106] reveal that free TAA molecules exist as two chiral conformers (D and L) having prismoid and propeller-like shape (Fig. 20c,d); the chiral states are dynamical, they can turn one into another. Further, it was established that these molecules, in principle, can form columnar filaments, which are stable (if long enough) at low TAA concentrations, $c>c^{*} \sim 10^{-8} \mathrm{M}$ due to $\pi$-stacking of aryl cores and $\mathrm{H}$-bonds between amide groups. It is important, however, that the conformation of TAA units in the columnar state must be nearly flat and less chiral (as compared to the free state) to ensure their correct $\pi$-stacking. Therefore, a transformation of TAA units in the association-ready state (flat and desolvated; cf. Figs. 22a and 20e,f) is

required for their columnar aggregation. Such molecular transformation is hindered by a significant activation energy $\left(E_{\mathrm{tr}} \sim 16 k_{B} T\right.$, cf. Fig. 22a) [106]. As a result, nucleation of columnar aggregates with neutral TAA units is totally prohibited 
kinetically.

There are two competing columnar structures: Stacking of units with similar chirality gives chiral columns (the so-called 'mercedez-benz' structure; Fig. 21a). On the other hand, TAA units with alternating propeller twirl sense tend to stack with $60^{\circ}$ rotation (so, the aryl rings of neighbouring TAA molecules recognize each other) to form achiral 'snowflake' columns (Fig. 21b). The latter structure is selected thermodynamically for TAA units of Fig. 20a. [106]

The results mentioned above imply that TAA units cannot aggregate in dilute solutions since filament nucleation there is arrested kinetically. This notion explains why TAA derivatives do not aggregate in the dark and in solvents like toluene or methanol. The remaining question, why the aggregation took place in chloroform under light irradiation, was addressed in refs. [114,106]. It was revealed that in the latter case the self-assembly is strongly facilitated by the presence of triarylammonium radical cations $\mathrm{TAA}^{+}$. The radical production is triggered by light-induced oxidation of TAAx molecules (excitation of TAAx by photon absorption followed by electron transfer from central amine, $\mathrm{N}$, to a chlorine of a neighboring chloroform molecule). A charge transfer complex $\left(\mathrm{TAA}^{+}+\mathrm{Cl}^{-}\right)$is formed as a result (Fig. 22b). The all-atomic modeling shows that radical cations are much more flat and less chiral than neutral TAAx molecules (Fig. 20e,f), so the cations tend to associate more readily in the columnar stacks. Moreover, this self-assembly is strongly favored by directional dipole-dipole interactions between the ions pairs, $\mathrm{TAA}^{+} \mathrm{Cl}^{-}$. As a result, the $\mathrm{TAA}^{+} \mathrm{Cl}^{-}$dipoles readily form the double-strand critical nuclei of the columnar structure (Fig. 22c) at low radical concentrations: the nuclei are further stabilized by $\pi-\pi$ and $\mathrm{H}$-bond interactions of radical cations. The columnar aggregation proliferates further by attachment of neutral TAAx molecules; at this stage the presence of radical cations is not required any more.

The kinetics of the radical-assisted association of TAAx units show some puzzling features $[114,106]$. For example, NMR and EPR data on continuously lightirradiated TAAx solutions reveal that saturation of radical amount in the filament aggregates takes about much (30 times) longer than their formation. This behavior was explained by the mechanism involving radical formation at the fibril ends and their slow diffusion inside the filament. [106]

It was also observed that aggregation of $\mathrm{TAAx}$ in chloroform solutions can be triggered by a tiny amount of radicals generated with a short light pulse, or a catalytic amount of added chemical oxidant or fibril seeds. [106] In this case no noticeable aggregation (monitored by time-resolved NMR) was detected for a considerable time (typically, for 100-1000s) after the triggering event. The aggregation then starts with an increasing rate followed by saturation at the level of nearly full transformation in the assembled state. The characteristic sigmoid aggregation curve is shown in Fig. 23. It was explained theoretically [106] with a 4-stage process (in the case of light or oxidant additive): (i) $\mathrm{TAA}^{-+}$radical production by photo-induced or chemical oxidation; (ii) critical fibril nuclei formation by radical cation/counterion pairs; (iii) growth and auto-catalytic self-replication of fibrils; (iv) saturation. During the most crucial 3rd stage the number of fibrils (initially defined by nucleation) increases in an exponential fashion due to their replication by fibril scission at the defect points. There are 3 types of scission events (the rate of each type of events is defined by the corresponding activation 
energy) [115]: (1) thermally activated equilibrium scission which is relevant for long enough fibril (whose length is comparable to the equilibrium fibril length); (2) scission at the trapped impurity defects (radical cation/counterion pairs occasionally entering the growing fibril); (3) scission at the kinetically trapped structural defects emerged during fibril growth. The total scission rate $\lambda_{\mathbf{s c}}$ is defined by the sum $(1)+(2)+(3)$ of all the contributions. The predicted theoretical curve for the fraction of aggregated TAAx is also shown in Fig. 23. The growth time $t_{\text {gr }}$ (stage iii) is defined by the scission rate, $t_{\mathbf{g r}} \propto\left(\lambda_{\mathbf{s c}}\right)^{-1 / 2}$; the lag-time (stage ii) is defined by $\lambda_{\text {sc }}$ and the nucleation rate which, in turn, strongly depends on the radical cation concentration.

Importantly, it was established [116-119] that the SA double-strand fibrils show high and strongly anisotropic metallic conductivity which can be attributed to a small amount of radical cations that are likely to dope the supramolecular filament structure. [106]

Another family of triarylamine-based molecules (TATA) is shown in Fig. 20b. The TATA molecules have similar generic structure (as TAA), but with 3 amide groups and 3 aliphatic tails per molecule. This functional core shows a strong ability to self-assemble in various solvents both in the presence of light or in the dark. [120,107]. TATA forms twisted single-columnar fibrils (Fig. 24) stabilized by $\pi$-stacking of aryl rings and 3 helical lines of $\mathrm{H}$-bonds between amide groups ('mercedez-benz' structure of Fig. 21a). After a long-time irradiation with white light of a chloroform solution of TATA, about $50 \%$ of TATA units get transformed in the radical cation state [107]. The irradiation heals the defects in the supramolecular structure (by their photo-induced excitation/elimination) and leads to a dramatic increase of the fibril conductivity [120]. In addition, the fibrils get positively charged (due to ammonium cations), but this charge is neutralized almost completely by the condensed counterions $\left(\mathrm{Cl}^{-}\right)$: the fraction of uncompensated charge is very low, $\sim 10^{-4}$. [107]

Surprisingly, it was also found [107] that TATA fibrils show a strong tendency to adsorb on the chloroform/water interface. These fibrils are practically neutral structures protected with hydrophobic alkyl tails. Why do they accumulate at the interface with water? The explanation is simple: although the condensed chlorides are strongly bound to the fibril (the $\mathrm{Cl}^{-} /$fibril bond energy $E_{b} \approx 22 k_{B} T$ ), the counterions prefer water environment so strongly (for both energy and entropy reasons) that about $1 / 3$ of them readily dissociate and go in the water phase once a fibril comes close to the interface. [107] The highly charged fibrils then get strongly attracted electrostatically to the 'image charge' in water (physically represented by the oppositely charged sublayer of anions in water). A highly anisotropic and dense positively charged interfacial monolayer of fibrils is formed as a result. Interestingly, such monolayer was used for precise alignment of plasmonic gold nanoparticles on the liquid/liquid (chloroform/water) interface: the citratecapped nanoparticles, initially dispersed in the water phase, get adsorbed on the positively charged layer of fibrils, and form an ordered 2-dimensional plasmonic structure there. [107] 


\section{Concluding remarks}

In this colloquium paper we discussed the impact of strongly-interacting chemical groups (SIGs) on self-assembly of amphiphilic molecules. Attraction of SIGs in solution naturally drives molecular aggregation which can result in ordered selfassembling (SA) structures rather than formation of a bulk condensed phase. The SA aggregates typically show a long-range positional packing order stemming from directional interactions of SIGs. However, a macroscopic propagation of the (periodic) ordered structure in the whole 3D space may be prevented by different frustration effects limiting the range of crystalline ordering and leading to finite dimensions of molecular aggregates in some directions. The simplest type of frustration is related to molecular amphiphilicity (that is, the presence of strongly solvophobic SIGs together with solvophilic chemical groups in the same molecule) inherent in all the SA systems considered herein. Frustration effects can be also related to geometrical mismatch between molecular elements (SIGs), orientational conflicts of their directional bonding interactions, and intrinsic molecular handedness (the presence of chiral centers). Numerous examples considered in this paper demonstrate that molecular 2D (layer) or 1D (filament) SA morphologies emerging in solutions of amphiphilic unimers can be defined by frustration effects due to the presence of SIGs.

In particular, we considered a number of systems with several types of disparate SIGs facilitating formation of SA structures ranging from lipid bilayers (serving as models for biomembranes whose complexity defines a wide range of functionalities in nature) and SA triarylamine nanowires (whose electrical properties can be controlled by light) stabilized by molecular amphiphilicity, to peptide twisted tapes and fibrils (resembling pathological amyloid plaques) whose stability originates, in particular, from the molecular chirality. In some cases the SA morphologies can be rationalized in terms of phenomenological theories involving continuous order parameter fields (like 2D or 3D nematic director or membrane surface curvature fields). This general approach is applicable if the order parameter functions are smooth enough (do not change much on the molecular length-scale), which is normally true for elastic bending in lipid bilayer tubules or similar morphologies with diameter $\sim 50-500 \mathrm{~nm}$ (see sections 4.1, 5.1, 5.2), but not for ripple bilayer structures or for defect points and lines (singularities) in the putative nematic director patterns of stripes in tilted lipid bilayers (section 5.1) involving length-scales comparable with molecular size. Moreover, the parameters involved in the continuum phenomenological theories (like intrinsic curvature of a SA layer, its elastic moduli for bending, Frank constants for the curl of nematic field or the cooperative degree of chirality) are also normally defined by short-range packing effects. In this regard, it is important to know precisely how the molecules pack together to form a locally ordered structure defined by short-range interactions of SIGs.

Frustrations in the packing molecular structure can also lead to a spontaneous symmetry breaking like spontaneous curvature of a SA membrane or its cooperative structural handedness. The interactions of SIGs and packing constraints are the key factors defining the spontaneous molecular tilt (cf. section 3), the spontaneously chiral helical structures of achiral molecules (cf. sections 5.2, 5.3), chiral selectivity in the DNA compaction transition (section 4.4) and other important phenomena. In particular, we surve a rare example of a theoretical study tracing the origin 
of a helical SA morphology of totally achiral amphiphilic molecules down to a submolecular level of packing structures of SIGs whose collective spontaneous chirality is elucidated with a simple physical mechanism (cf. section 5.2). More generally, the spontaneous chirality induction in SA systems of achiral molecules can be attributed to such major driving factors as the anisotropic packing effects, directional bonds, geometrical constraints and enantiomeric conformers (rotational isomers) of chemically achiral 'blocks' like molecular 'propellers' capable of forming helical filaments (cf. sections 5.3 and 5.1).

In spite of the reviewed progress in understanding the role of SIGs in amphiphilic structures, new efforts (atomistic modeling in tandem with continuum theory development) are invited to unveil the basic relationships between the unimer nature and the emerging self-assembly morphologies.

\section{Acknowledgements}

We acknowledge fruitful discussions with A.R.Khokhlov, J.-F.Joanny, N.Boden, A.Aggeli, P.Mesini, A.Johner and J.Baschnagel. A partial support from the IRTG 'Soft Matter Science' is acknowledged as well. 


\section{References}

[1] Doi M., Edwards S.F., The Theory of Polymer Dynamics (Clarendon, Oxford) 1986.

[2] De Gennes, P.-G. Scaling Concepts in Polymer Physics; Cornell Univ. Press: Ithaca, 1979.

[3] A. Grosberg, A. Khokhlov, Statistical Physics of Macromolecules, American Institute of Physics, New York, 1994.

[4] M. Rubinstein, R.H. Colby, Polymer Physics, Oxford University Press, Oxford, UK, 2003.

[5] P.G. de Gennes, J. Prost, The Physics of Liquid Crystals, Clarendon Press, Oxford, 1998.

[6] L.D.Landau, E.M.Lifshitz, Statistical Physics (Pergamon Press, Oxford, 1998).

[7] A.N.Semenov, Phys. Rev. E 73, 041803 (2006).

[8] De Gennes P.G., Adv. Colloid. Interface Sci., 1987, v.27, 189.

[9] Nyrkova,I.A.; Khokhlov,A.R. and Doi,M. Macromolecules 1993, 26, 3601.

[10] A. N. Semenov, I. A. Nyrkova and A. R. Khokhlov, Macromolecules, 1995, $28,7491-7500$.

[11] A.N.Semenov, I.A.Nyrkova, A.R.Khokhlov Statistics and dynamics of ionomers. In: 'Ionomers: Characterization, Theory and Applications', S.Schlick, ed., CRC Press, 1996, pp.251-279.

[12] Folkes, M.J., Ed. / Processing, Structure and Properties of Block-Copolymers; Elsevier: New York, 1985.

[13] Goodman, I., Ed. / Developments in Block Copolymers; Applied Science Publishers: New York, 1982 (Vol.1), 1985 (Vol.2).

[14] Helfand, E.; Wasserman, Z.R. Macromolecules, 1980, v.13, p.994.

[15] L. Leibler, Macromolecules 13, 1602 (1980).

[16] A.N. Semenov, Sov.Phys.JETP 61, $733 \quad$ (1985). [A.N.Semenov, Zh.Eksp.Teor.Fiz. 88, 1242 (1985)].

[17] Fredrickson, G.H.; Helfand, E. J.Chem.Phys., 1987, v.87, p.697.

[18] Semenov, A.N. Macromolecules, 1989, v.22, p.2849.

[19] Williams,C.E. In: Multiphase Macromolecular Systems; Culbertson,B.M., Ed.; Plenum Press: New York, 1989; Vol. 6.

[20] Gouin,J.P.; Williams,C.E. and Eisenberg,A. Macromolecules 1989, 22, 4573.

[21] Lantman, C.W.; MacKnight, W.J.; Lundberg, R.D. / In: Comprehensive Polymer Science; Allen, G.; Bevington, J.C., Eds.; Pergamon Press: Oxford, 1989, Vol.2, Chapter 25.

[22] Moore,R.B.; Bittencourt,D.; Gauthier,M.; Williams,C.E. and Eisenberg,A. Macromolecules 1991, 24, 1376.

[23] Daoud, M.; Cotton, J. P. J. Phys. 1982,43, 531.

[24] Witten, T. A.; Pincus, P. A. Macromolecules 1986,19, 2509.

[25] Birshtein,T.M.; Zhulina,E.B. Polymer 1989, v.30, 170.

[26] P.G.Khalatur, A.R.Khokhlov, I.A.Nyrkova, A.N.Semenov, Macromol.Theory Simul. 5, 713-747 (1996).

[27] P.G.Khalatur, A.R.Khokhlov, I.A.Nyrkova, A.N.Semenov, Macromol.Theory Simul. 5, 749-757 (1996).

[28] Rebrov,A.V.; Ozerin,A.N.; Svergun,D.I.; Bobrova,L.P.; Bakeev,N.F. Vysokomol.Soedin. 1990, v.32A, 1593.

[29] Hilger,C; Stadler,R. Macromolecules 1992, v.25, 6670. 
[30] Lu,X.; Steckle,W.P.,Jr.; Weiss,R.A. Macromolecules 1993, v.26, 6525.

[31] Kim,.J.-S.; Jackman,J.; Eisenberg,A. Macromolecules 1994, v.27, 2789.

[32] S.J.Holder, N.A.J.M.Sommerdijk, Polym.Chem. 2, 1018 (2011).

[33] 67 A. Ramzi, M. Prager, D. Richter, V. Efstratiadis, N. Hadjichristidis, R. N. Young and J. B. Allgaier, Macromolecules, 1997, 30, 7171- 7182.

[34] W. Wang, R. Liu, Z. Li, C. Meng, Q. Wu and F. Zhu, Macromol. Chem. Phys., 2010, 211, 1452-1459.

[35] A. Constancis, R. Meyrueix, N. Bryson, S. Huille, J. M. Grosselin, T. Gulik-Krzywicki and G. Soula, J. Colloid Interface Sci., 1999, 217, 357-368.

[36] I. K. Voets, A. de Keizer, P. de Waard, P. M. Frederik, P. H. H. Bomans, H. Schmalz, A. Walther, S. M. King, F. A. M. Leermakers and M. A. Cohen Stuart, Angew. Chem., Int. Ed., 2006, 45, 6673-6676.

[37] Gregory M. Grason, J. Chem. Phys. 145, 110901 (2016); doi: $10.1063 / 1.4962629$

[38] Jean-François Sadoc, Rémy Mosseri, Geometrical Frustration. Cambridge, UK: Cambridge University Press, October 1999. ISBN 0521441986.

[39] R.B.Gennis, Biomembranes, Springer-Verlag, Berlin, 1989.

[40] S. Karaborni, S. Toxvaerd, J.Chem.Phys. 97, 5876 (1992).

[41] A.N.Semenov, Mol.Cryst.Liq.Cryst. 209, 191 (1991).

[42] Stevens, Peter S. Patterns in Nature. Little, Brown \& Co. (1974).

[43] A. Jamal, I. Nyrkova, Ph. Mesini, S. Militzer, G. Reiter, Nanoscale 9, 3293 (2017).

[44] G. W. Ehrenstein; Richard P. Theriault (2001). Polymeric materials: structure, properties, applications. Hanser Verlag. ISBN 1-56990-310-7.

[45] M.Wintermantel, K.Fischer, M.Gerle et al. Angew.Chem. 34, 1472 (1995).

[46] A.V.Subbotin, A.N.Semenov, Polym. Sci. A 49, 1328 (2007).

[47] T. Nakano and Y. Okamoto, Chem. Rev. 2001, v.101, pp.4013-4038.

[48] K.Akagi Chem. Rev. 2009, v.109, pp.5354-5401.

[49] K.Akagi, G.Piao, S.Kaneko et al. Science 282, 1683 (1998).

[50] Green, M. M.; Park, J.-W.; Sato, T.; Teramoto, A.; Lifson, S.; Selinger, R. L. B.; Selinger, J. V. Angew. Chem., Int. Ed. 1999, 39, pp.3138-3154.

[51] J.V.Selinger, M.S.Spector, J.M.Schur, J.Phys.Chem.B 105, 7157 (2001).

[52] J.M.Schnur, Science 262, 1669 (1993).

[53] J.M.Schnur, R.Shashidhar, Adv.Mater. 6, 971 (1994).

[54] R.Oda, I.Huc, S.J.Candau, Angew.Chem. 37, 2689 (1998).

[55] R.Oda, M.Schmutz, S.J.Candau, F.C.Mackintosh, Nature 399, 566 (1999).

[56] I.A. Nyrkova, A.N. Semenov, Soft Matter 6, 501 (2010).

[57] R.Lipowsky, Nature 349, 475 (1991).

[58] W.Helfrich, J.Prost, Phys.Rev.A 38, 3065 (1988).

[59] D.S.Chung, G.B.Benedek, F.M.Konikoff, J.M.Donovan, PNAS 90, 11341 (1993).

[60] Z.C.Tu, U.Seifert, Phys.Rev.E 76, 031603 (2007).

[61] Y. Wang, D. Zhou, H. Li, R. Li, Y. Zhong, X. Sun and X. Sun, J. Mater. Chem. C 2, 6402 (2014).

[62] M.S.Spector, V.J.Selinger, A.Singh, J.M.Rodriguez, R.R.Price, J.M.Schnur, Langmuir 1999, v.14, pp.3493-3500.

[63] A.Brizard, C.Aim, T.Labrot, I.Huc, D.Berthier, F.Artzner, B.Desbat, R.Oda, J.Am.Chem.Soc. 129, 3754 (2007). 
[64] W.Si et al. Tetrahedron Lett. 52, 2484 (2011).

[65] D.Berthier, T.Buffeteau, J.-M.Leger, R.Oda, I.Huc, J.Am.Chem.Soc. 124, 13486 (2002).

[66] R.Oda, F.Artzner, M.Laguerre, I.Huc, J.Am.Chem.Soc. 130, 14705 (2008).

[67] D.G.Rhodes et al. Chem.Phys.Lipids 49, 39 (1988).

[68] J.B.Lando, R.V.Sudiwala, Chem.Mater. 2, 594 (1990).

[69] M.Caffrey, J.Hogan, A.S.Rudolph, Biochemistry 30, 2134 (1991).

[70] E.Sackmann, A.Fischer, W.Frey, in Physics of Amphiphilic Layers, Springer, Berlin, 1987.

[71] D.R.Nelson, L.Peliti, J.Physique 48, 1085 (1987).

[72] H.S.Seung, D.R.Nelson, Phys.Rev.A 381005 (1988).

[73] B.N.Thomas et al. Science 267, 1635 (1995).

[74] A.Aggeli, M.Bell, N.Boden, et al. Nature 386, 259 (1997).

[75] I.A.Nyrkova, A.N.Semenov, A.Aggeli, N.Boden, European Physical Journal B, 2000, v.17, No.3, pp.481-497.

[76] A.Aggeli, M.Bell, N.Boden, J.N.Keen, T.C.B. McLeish, I.Nyrkova, S.E.Radford, A.Semenov, J.Material Chem., 1997, v.7, No.7, pp.1135-1145.

[77] A.Aggeli, M.Bell, N.Boden, R.Harding, T.C.B.McLeish, I.Nyrkova, S.E.Radford, A.Semenov, The Biochemist, 2000, v.22, pp.10-14.

[78] I.A.Nyrkova, A.N.Semenov, A.Aggeli, M.Bell, N.Boden, T.C.B.McLeish, European Physical Journal B, 2000, v.17, No.3, pp.499-513.

[79] A.Aggeli, I.A.Nyrkova, M.Bell, R.Harding, L.Carrick, T.C.B.McLeish, A.N.Semenov, N.Boden, PNAS, 2001, v.98, p.11857-11862.

[80] A.Aggeli A, I.A.Nyrkova, M.Bell, L.Carrick, T.C.B.McLeish, A.N.Semenov, N.Boden, 'Exploiting peptide self-assembly to engineer novel biopolymers: tapes, ribbons, fibrils and fibres'. In: 'Self-assembling Peptide Systems in Biology Medicine and Engineering' (A. Aggeli, N. Boden, S, Zhang, Eds.), Kluwer Acad. Publ., 2001, pp.1-17.

[81] R.P.W.Davies, A.Aggeli, A.J.Beevers, N.Boden, L.M.Carrick, C.W.G.Fishwick, T.C.B.McLeish, I.A.Nyrkova, A.N.Semenov, Supramolecular Chemistry 2006, v.18 No5: pp.435-443.

[82] Davies RPW, Liu B, Maude S, Carrick LM, Irina Nyrkova, Tom C. McLeish, Sarah A. Harris, Biopolymers e23073 (2017). https://doi.org/10.1002/bip.23073

[83] Aggeli A.; Bell M.; Boden N.; Carrick L.; Harding R.; McLeish T.C.B.; Nyrkova, I.A.; Semenov A.N., 'Impact of chirality on one-dimensional selfassembling systems', In: 'Self-Assembly', pp.92-104 , 2003 , IOS Press.

[84] Cohen F.E., J.Molec.Biology, 1999, v.293, 313.

[85] Tan S.Y., Pepys M.B., Amyloidosis. Histopathology, 1994, v.25, 403.

[86] C.F.Jordan, L.S.Lerman, J.H.Venable, Nature (1972) 236, 67.

[87] A.A.Zinchenko, V.G.Sergeyev, V.A.Kabanov, S.Murata, K.Yoshikawa, Angew.Chem.Int.Ed. (2004) 43, 2377.

[88] A.A.Zinchenko, N.Chen, S.Murata, K.Yoshikawa, ChemBioChem (2005) 6, 1419.

[89] I.A.Nyrkova, A.N.Semenov, Soft Matter 5, 979 (2009).

[90] S.Wang, Y.Zhang, Y.Xia, B.Song, Nanoscale 7, 17848 (2015).

[91] Y. Wang, D. Zhou, H. Li, R. Li, Y. Zhong, X. Sun and X. Sun, J. Mater. Chem. C 2, 6402 (2014). 
[92] J. Kim, J. Lee, W.Y. Kim, H. Kim, S. Lee, H.C. Lee, Y.S. Lee, M. Seo and S.Y. Kim, Nat. Commun. 6, 6959 (2015).

[93] Anuradha, D.D. La, M. Al Kobaisi and S.V. Bhosale, Sci. Rep. 5, 15652 (2015).

[94] B. Song, B. Liu, Y. Jin, X. He, D. Tang, G. Wu, S. Yin, Nanoscale 7, 930 (2015).

[95] W. Yang, X. Chai, L. Chi, X. Liu, Y. Cao, R. Lu, Y. Jiang, X. Tang, H. Fuchs, T. Li, Chem. Eur. J. 5, 1144 (1999).

[96] J.M. Ribo, J. Crusats, F. Sagues, J. Claret, R. Rubires, Science 292, 2063 (2001).

[97] S. Pakhomov, R.P. Hammer, B.K. Mishra, B.N. Thomas, PNAS 100, 3050 (2003).

[98] U. Seifert, J. Shillcock, P. Nelson, Phys.Rev.Lett. 77, 5237 (1996).

[99] J.V. Selinger, Z.-G. Wang, R.F. Bruinsma and C.M. Knobler, Phys. Rev. Lett. 70, 1139 (1993).

[100] J.V.Selinger, J.M.Schnur, Phys. Rev. Lett. 71, 4091 (1993).

[101] J.V.Selinger, F.C.MacKintosh, J.M.Schnur, Phys.Rev.E 53, 3804 (1996).

[102] R.Koynova, M.Caffrey, Biophys.Acta 1376, 91 (1998).

[103] O.Lenz, F.Schmid, PRL 98,058104 (2007).

[104] A.H.de Vries, S.Yefimov, A.E.Mark, S.J.Marrink, PNAS 102, 5392 (2005).

[105] A.Singh et al. Chem.Phys.Lipids 47, 135 (1988).

[106] I. Nyrkova, E. Moulin, J.J. Armao IV, M. Maaloum, B. Heinrich, M. Rawiso, F. Niess, J.-J. Cid, N.Jouault, E. Buhler, A.N. Semenov, and N. Giuseppone, ACS Nano 8, 10111 (2014).

[107] J.J. Armao IV, I. Nyrkova, G. Fuks, A. Osypenko, M. Maaloum, E. Moulin, R. Arenal, O. Gavat, A. Semenov, and N. Giuseppone, J. Am. Chem. Soc. 139, 2345 (2017).

[108] D.M. Bassani, J.-M. Lehn, G. Baum, D. Fenske, Angew. Chem. Int. Ed. 36, 1845 (1997).

[109] N. Diaz, F.-X. Simon, M. Schmutz, M. Rawiso, G. Decher, J. Jestin, P.J. Mesini, Angew. Chem. 44, 3260 (2005).

[110] N. Diaz, F.X. Simon, M. Schmutz, P. Mesini, Macromol. Symp. 241, 68 (2006).

[111] F.X. Simon, T.T. Tam Nguyen, N. Diaz, M. Schmutz, B. Deme, J. Jestin, J. Combet, P.J. Mesini, Soft Matter 9, 8483 (2013).

[112] I.A.Nyrkova, A.N.Semenov, Polymer (2018, in press).

[113] Y.Shirota, H.Kageyama, Chem.Rev. 107, 953 (2007)

[114] Moulin, E.; Niess, F.; Maaloum, M.; Buhler, E.; Nyrkova, I.; Giuseppone, N., Angew. Chem. Int. Ed. 2010, 49, 6974-6978.

[115] I.A.Nyrkova, A.N.Semenov, The European Physical Journal E, 2007, v. 24, no.2, pp.167-183.

[116] Moulin, E.; Niess, F.; Jouault, N.; Buhler, E.; Giuseppone, N., Nanoscale 2012, 4, 6748-6751.

[117] Faramarzi, V.; Niess, F.; Moulin, E.; Maaloum, M.; Dayen, J.-F.; Beaufrand, J.-B.; Zanettini, S.; Doudin, B.; Giuseppone, N., Nature Chemistry 2012, 4, 485-490.

[118] Moulin, E.; Martin, J.-J.; Giuseppone, N., Adv. Mater. 2013, 25, 477-487.

[119] Giuseppone, N., Acc. Chem. Res. 2012, 45, 2178-2188. 
[120] Armao, J.J.; Maaloum, M.; Ellis, T.; Fuks, G.; Rawiso, M.; Moulin, E.; Giuseppone, N., J. Am. Chem. Soc. 136, 11382 (2014), dx.doi.org/10.1021/ja5044006.

\section{FIGURE CAPTIONS}

FIG. 1. (a) Phase diagram of a melt of AB diblock copolymers with geometrically similar $\mathrm{A}$ and $\mathrm{B}$ units $[15,16] . \Phi_{A}$ is volume fraction of A-component; $\chi$, the Flory interaction parameter; $N$, the total polymerization index. Dis - disordered phase, BCC - microphase with spherical domains, Hex - superstructure with cylindrical domains, Lam - lamellar phase. (b) Diblock copolymer AB; $N_{A}, N_{B}$ are the polymerization indices of the A, B blocks, respectively. (c) Multi-block copolymer $(\mathrm{AB})_{6}$. (d) Lamellar phase (Lam). (e) Cylindrical microdomains (Hex). (f) Superstructure of spherical domains (BCC).

FIG. 2. Micelles of asymmetric multi-block-copolymers, $V_{B} \gg V_{A}$ : (a) Spherical micelle: an A-core of radius $R$ is surrounded by a corona of B-blocks (loops). In the superstrong segregation limit $R$ tends to $R_{\max }=L_{A} / 2$. The size of micellar corona, $R_{2}$, is much larger than $R$. (b) A cylindrical aggregate with core of length $D$ and radius $R_{\max }=H / 2$. (c) Aggregate with disklike A-core of total diameter $D$ and thickness $H=2 R_{\max }=L_{A}$. (d) The B-corona stays nearly spherical if $R_{2} \gg D$. (e) Unilamellar vesicle.

FIG. 3. The schematic scaling diagram of block-copolymer micelle morphologies using log-log scale in variables: the reduced core-surface tension $\tilde{\sigma}=\sigma s_{A} /\left(k_{B} T\right)$ vs. copolymer composition $x=V_{A} / V_{B}=L_{A} s_{A} /\left(L_{B} s_{B}\right)$. The vertical scale is based on the parameter $z=\sqrt{\frac{L_{B}}{l_{B}}} \frac{s_{B}}{s_{A}} ; x_{A} \sim \frac{l_{A} s_{A}}{L_{B} s_{B}}$ corresponds to $L_{A} \sim l_{A}$. The transition lines are drawn with scaling accuracy (neglecting numerical and logarithmic factors): (1) critical line of micelle formation; (2) continuous (second order) transition from spheres to disks at $\sigma=\sigma^{*} ;(3)$ discontinuous (first order) transition from disks to infinite lamellae at $\sigma=\sigma^{* *}$.

FIG. 4. (a) Head-rod amphiphilic molecule with head area $s_{h}$, rod length $L_{r}$ and its cross-section area $s_{r}$; (b) untilted monolayer or bilayer phase ( $\operatorname{Sm} A$ ) with rods normal to the layer; (c) tilted phase $(\mathrm{Sm} C)$. The tilt angle is $\psi$, tilt director $\underline{m} ; \underline{m}_{1}$ and $\underline{m}_{2}$ are the directors of the two opposite sublayers.

FIG. 5. (a) Rod-coil copolymer; $L_{t}$ is the contour length of a flexible tail, $l_{t}$ is its Kuhn segment, $s_{t}$, its cross-section $\left(L_{r}, s_{r}\right.$ are the rod length and cross-section parameters). (b) Nematic phase $(N)$. (c,d) Smectic A phases. (e,f) Smectic C phases.

FIG. 6. Phase diagram of rod-coil copolymer melt [41] in the coordinates $\lambda=V_{t} / V_{r}$ vs. $\tilde{\chi}=\chi L_{r} s_{r} / v_{t}$ (in logarithmic scale) showing nematic $(N)$, smectic $\mathrm{A}\left(A_{1}\right.$, $\left.A_{2}\right)$ and smectic $\mathrm{C}\left(C_{1}, C_{2}\right)$ phases. Solid and dashed lines show 1st-order and 2nd-order phase transitions, respectively. Line 1: $\lambda \leq 1, \tilde{\chi}_{1} \simeq 4.9(1+2 \nu / \lambda)$; line $4: \quad \lambda \geq 1, \tilde{\chi}_{4} \sim \lambda^{1 / 3} ; \nu=l_{t} s_{r} /\left(6 L_{r} s_{t}\right) \ll 1$.

FIG. 7. (a) Schematic structure of dumbbell-like A-B-A molecule: 'A', 'B' are rigid SIGs connected by flexible spacers. (b) Uniform layer structure of self-assembled A-B-A molecules. (c) The molecular layer with a pattern of A-ribbons. 
The schematic pattern of nanoribbons observed in a cyclohexanone solution of BHPB10 [43], with $n$ A-groups across the ribbon. (e) Pattern of stripes in the layer of A-B dumbbell molecules adsorbed on a solid support.

FIG. 8. (a) Comb-like macromolecule: backbone with repeat units of length $b$ and side moieties of size $B$; the spacer length is $r-B / 2$. (b) A perfect helical fragment. (c) A defect (red arrow) where the helical sense changes sign. (d) Twisted comb-like molecule with long L- and D-helical fragments.

FIG. 9. (a) Twisted ribbon with pitch $P$ and width $W$; (b) helical ribbon. [56]

FIG. 10. Fibril formation in solutions of identical oligo-peptides. [75] Antiparallel $\beta$-sheet of stretched peptide chains modeled as rods (a) and its representation as a tape (b); $\underline{n}$ is unit vector locally normal to the tape, $\underline{t}$ is parallel to the tape. Face-to-face attraction between the tapes (c) could lead to their infinite stacks (2D-sheets) (d) if the primary building unit had a cuboid shape (e). Self-assembly of twisted cuboids (f) reflecting the handedness of natural aminoacids leads to a twisted tape $(\mathrm{g})$. A fibril made of a few stacked tapes (h) whose axis lines are entwined (i). $h_{0}$ is the twist pitch of the individual tape, $h$ is the fibril twist period; $b$ is tape width, $a$ is thickness per tape in the stack.

FIG. 11. Phase diagram of peptide solution in variables $\tilde{k}=k_{\text {bend }} / k_{\text {twist }}, \quad \tilde{\sigma}=$ $\sigma a b h_{0} / k_{\text {twist }} ; \tilde{a}=a / h_{0} \lesssim 1$. [75,79] The lines of phase transitions are shown in red; the first-order transition from lone tapes to fibrils is marked with $\tilde{\sigma}^{*}$, and the second order transition from fibrils to infinite stacks is marked with $\tilde{\sigma}^{* *}$. Blue dotted lines mark the crossovers between various fibril regimes: thin fibrils with low, moderate and high bending moduli (LB, MB and $\mathrm{HB}$, respectively) and wide fibrils (WF).

FIG. 12. The hierarchical SA structures (c-f) in solutions of peptide chiral molecules (a,b) emerging as their concentration is increased. [79] Chiral unimer should be activated with energy $E_{\mathrm{tr}}$ (b) to turn rod-like and to allow for supramolecular assembly of unimers (a) into tapes (c) with correct recognition of complementary groups. The twisted tape (c) has distinct faces ('red' and 'grey'), the 'red' face is hydrophobic. Attraction between 'red' faces results in formation of double tapes (ribbons) (d) having identical 'grey' faces. An attraction of weaklyhydrophobic 'grey' faces of the ribbons leads to fibril formation (e). Finally, attraction between yellow ends of the rods results in fibril stacking yielding fibres (f). The fibre shown in (f) involve two entwined fibrils (pink and violet).

FIG. 13. Expanded coil of a strongly-charged DNA (a) and its globular state (b) condensed by adding a low-molecular salt (multivalent counterions). Designed chiral divalent cations: left-handed SS2+ (c) and right-handed RR2+ (d) enantiomers used to induce the chirality-sensitive DNA collapse $[87,88]$ in vitro. (e): The experimental (symbols) and theoretical (solid lines) dependencies of the fraction of coiled DNA, $f_{c}$, on the total dication concentration $c$ (in $\mathrm{M}=\mathrm{mol} / \mathrm{L}$ ) for pure SS2+ and RR2+ enantiomers and for their mixtures. Experimental data [87]: $\mathrm{x}$ for SS, $\square$ for RR, $\diamond$ for racemic mixture. The theory is based on the 'clinch/bridge' competition model [89]; blue, violet, red and green curves 
correspond to $0 \%, 5 \%, 50 \%$ and $100 \%$ of RR2+ enantiomer. (Inset: the relative values of association energies for 'clinches' (adsorption of SS2+ and $\mathrm{RR} 2+$ ) and for bridges having the same energy for both enantiomers). (f): An RR2+ dication (shown in green) is bound to the DNA minor groove. (Inset: expanded view of the dication with H-bonds shown; the H-atoms involved in the bonding are shown in green; the corresponding O-atoms are green with red centers. (g): Dication 'bridges' between 2 parallel DNA fragments with the optimum separation $h^{*}$ between their surfaces; the bridges compete with dication adsorption in the minor groove of each DNA fibril.

FIG. 14. A model of a tilted lipid bilayer: side view (a) and top view (b) with optimal antiparallel orientations of tilt directors $\underline{m}_{1}, \underline{m}_{2}$ in the constituent monolayers. A more general situation with the angle $\pi-\alpha$ between the directors (c). (d): A diagram showing the directors $\underline{m}_{1}, \underline{m}_{2}$, the median axis (the black line) and the principal curvature axis 1 (pink line 'p.a.1'); $\phi$ is the angle between the principal axis 1 and the median.

FIG. 15. Diamide-ester molecules and the membranes formed by them in cyclohexane. [112] The chemical (a) and schematic (b) structures of BHPB-n molecules (the ester-tail length $n$ was varied from 5 to 16). Monolayer (c) and bilayer (d) membrane structures of BHPB molecules with amide groups (red), aromatic rings (green) and hydrocarbon spacers (blue). The optimal structures of individual sublayers of SIGs stabilizing the membranes as obtained by all-atomic modeling: the sublayers of aromatic rings $(e, g)$ and of amide groups $(f, h)$ in the monolayer $(e, f)$ and in the bilayer $(g, h)$ membranes (two perpendicular views along the sublayers are shown for each case). The specific areas per SIG are: $S_{\text {ring }} \approx 30.4 \AA^{2}$ in the sublayers of rings $(\mathrm{e}, \mathrm{g})$, while in the sublayers of amide groups $S_{\text {am }}=S_{1} / 2 \approx 17.1 \AA^{2}$ for monolayer membrane (f) and $S_{\text {am }}=S_{2} \approx 33.5 \AA^{2}$ for bilayer membrane $(\mathrm{h})$.

FIG. 16. (a) Ring sublayer dilution (an increase of the inter-columnar distance $d$ ) leads to a larger area per aromatic ring, $S_{\text {ring }}^{\text {dil }}=\left(1+d / D_{c}\right) S_{\text {ring }}$, allowing to match the specific areas per molecule in different sublayers of the same membrane. (b) Fragmentation and splitting of a membrane in stripes.

FIG. 17. Spontaneous chiral symmetry breaking in BHPB membranes [112]. (a): The amide and the ring SIGs connected with alkyl spacers in a fragment of BHPB molecule (front view). The CO-NH dipoles (shown as blue arrows) are not parallel to the sublayers in the single-molecule ground state. (b): The same fragment after a left-hand twisting of the spacers 'III' in order to render the $\mathrm{CO}-\mathrm{NH}$ dipoles parallel to the sublayers (side view). (c): Top view of the ring and amide sublayers forming the bilayer (cf. Fig. $15 \mathrm{~d}$ ), $\tilde{\alpha} \approx 25^{0}$, rings are shown as green disks, lines of H-bonds are shown as blue dashed lines (only the bottom amide sublayer is shown). (d): The same fragment (as in part (a)) after twisting the spacers 'III' in order to achieve the vertical orientation of the amide groups (in accordance with the monolayer membrane structure, Fig. 15f). (e): Top view of the amide sublayer within the monolayer structure, Fig. 15c. The ring 'II' and two spacers 'III' of one BHPB molecule (shown in (d)) are marked in yellow color, the ring plane is shown by green dashed line, the amide groups of this molecule are surrounded with red and 
pink ovals. The line of H-bonds is shown with blue dashed line, $\alpha \approx 60^{\circ}$. (f): The monolayer membrane structures resulting from twisting the spacer in two alternative directions.

FIG. 18. Schematic picture of the cylindrically curved monolayer formed by BHPB molecules due to SIG area mismatch [112]. (a): The direction of the global cylindrical axis $y$ is shown with black arrows. The ring sublayer (green) consists of stacked rings (shown as hexagonal columns parallel to the $y$-axis). The amide sublayer is shown in red, alkyl spacers in blue, the lines of H-bonds between amide groups are white. (b): The globally tubular shape of the same curved monolayer; $R$ is the mean radius of cylindrical curvature, $h_{\text {tot }}$ is the wall thickness.

FIG. 19. The predicted high aspect ratio morphologies of monolayer BHPB aggregates [112]. (a) The right-handed helical structure: side and 3D views. Black arrows show the cylinder axis and simultaneously the direction of $\pi$ - $\pi$ stacked columns of aromatic SIGs. Green rings correspond to the aromatic sublayer (rings instead of continuous cylinder are shown for clarity). Red and blue helices show the direction of lines of $\mathrm{H}$-bonds stabilizing the amide sublayer $(2$ colors are used for clarity); the helical lines form an angle $\theta=90^{\circ}-\alpha \approx 30^{\circ}$ with the cylinder axis. (b-d) The main 3D morphologies of the BHPB monolayers: helical ribbon (b), tubule (c) and hollow tube (d). All objects have the same curvature radius $R$, the same chirality (defined by the angle $\theta$ ) and the same helical pitch $H_{p}$, but they differ in the ribbon width $D=\left(H_{p}-b\right) \sin \theta$. (e) The diagram of $3 \mathrm{D}$ morphologies: edge energy parameter $\widetilde{\Gamma} \equiv(\Gamma-1) \times 10^{\mathbf{3}}$ vs. reduced curvature $\widetilde{C} \equiv h / R$, where $\Gamma \equiv\left|\gamma_{t}\right| / \gamma_{\text {SIG }} \propto n^{2} / n^{* 2}$, $h$ is related to the membrane thickness.

FIG. 20. (a,b): Generic chemical structure of photosensitive self-assembling triarylamine (TAA) molecules: (a) triarylamine-amide molecular units (TAAx) [114] and (b) tris-amide triarylamine (TATA) units [120]; the TAA (triphenylamine) SIGs are marked with yellow circles, the amide SIGs - with pink ovals and the tails enhancing molecular solubility - with cyan circles; $R_{1}$ can be oligo-alkyl or benzene, $R_{3}$ is either $\mathrm{H}$ or $\mathrm{Cl}$, and $R_{y}$ is an alkyl tail $C_{n} H_{2 n+1} \quad(n=1-18)$. (c-f): The prismoid propeller-like shape of TAA unit in the ground state: (c) prismoid geometry of the $N C_{3}$ molecular core center $\left(\delta_{i} \simeq 10^{0}-13^{0}\right)$, and (d) propeller-like conformation of the phenyl rings in neutral TAA $\left(\phi \simeq 40^{\circ}-60^{\circ}\right.$ depending on the nature of the substituents $\left.R_{i}\right)$; the L-conformer is shown. D(e) and L- (f) enantiomeric conformers of a photo-excited TAA molecule (or a radical $\left.\mathrm{TAA}^{+}\right)$with nearly flat propeller-like shape $\left(\phi \simeq 30^{\circ}-45^{0}, \delta \simeq 0^{0}\right)$.

FIG. 21. (a) Mercedez-benz chiral structure; (b) snowflake structure [106].

FIG. 22. (a): Activation is needed to transform a TAA unimer from initial prismoid to the association-ready flat state. (b): Photoxidation in chloroform causes formation of stable charge-transfer $\mathrm{TAA}^{+} \mathrm{Cl}^{-}$ion pairs. (c): $\mathrm{TAA}^{+} \mathrm{Cl}^{-}$ pairs aggregate to form a critical nucleus (involving $n^{*}=6$ radical cations) of the double columnar snowflake structure. [106]

FIG. 23. Autocatalytic fibrillization of fresh monomeric solution of TAAx $(10 \mathrm{mM})$ in chloroform. The fraction $w$ of self-assembled TAAx vs. time $t$ after adding a 
chemical oxidant with mole fractions (from left to right) $=5,1,0.5,0.1,0.01 \%$ : NMR data (symbols) and theory curves (lines) [106].

FIG. 24. The TATA twisted fibril [107]. 

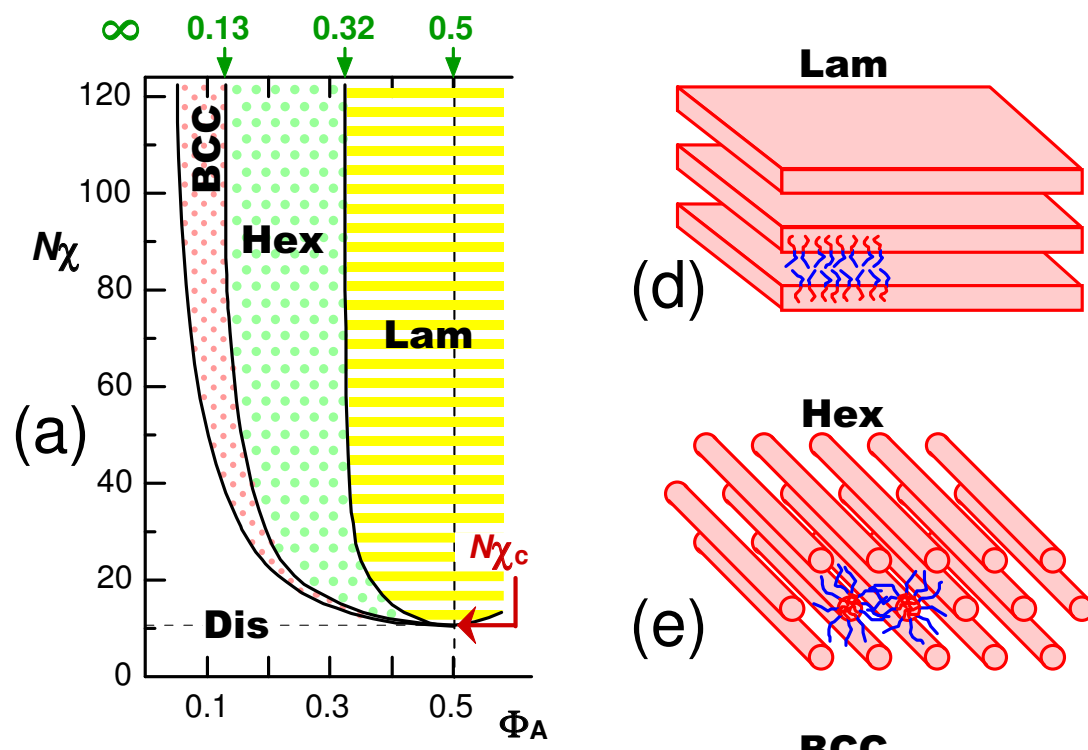

(b) $\overbrace{}^{N_{A}} \sim \sim^{N_{B}}$

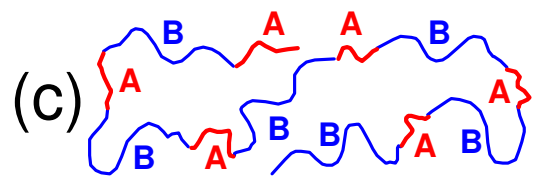
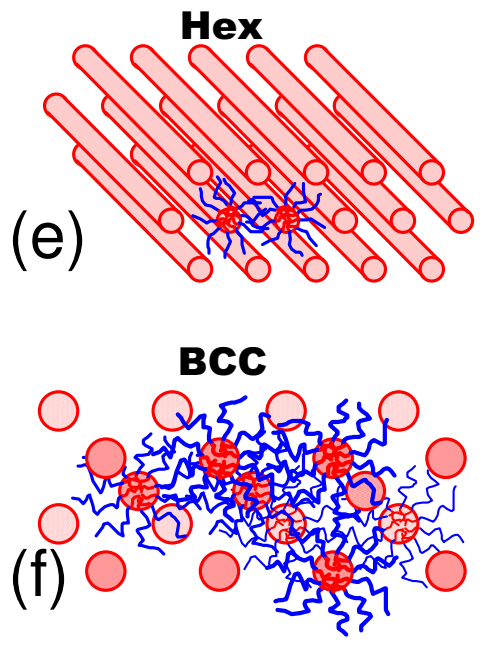

Fig.1

(a)
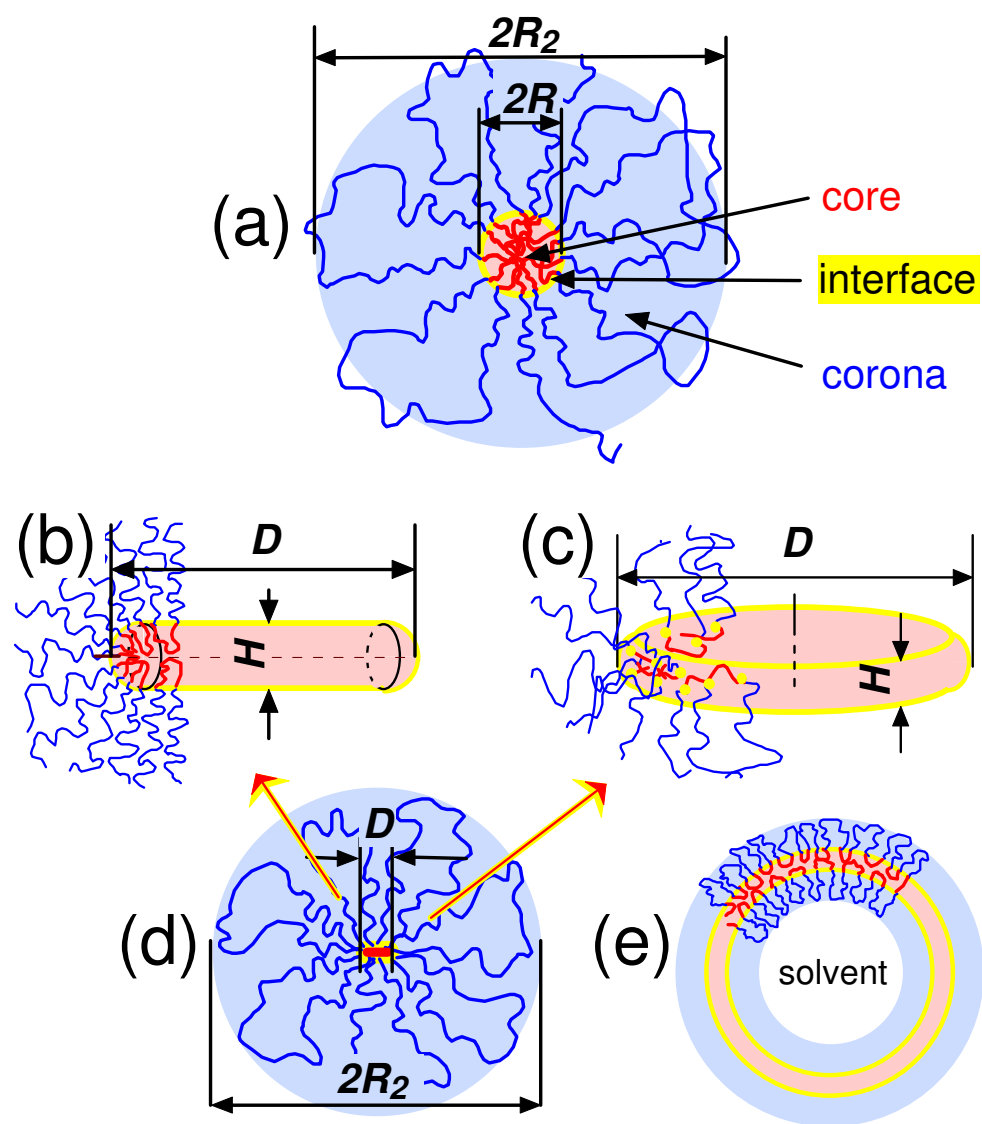

(e)

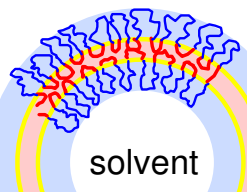

Fig. 2 


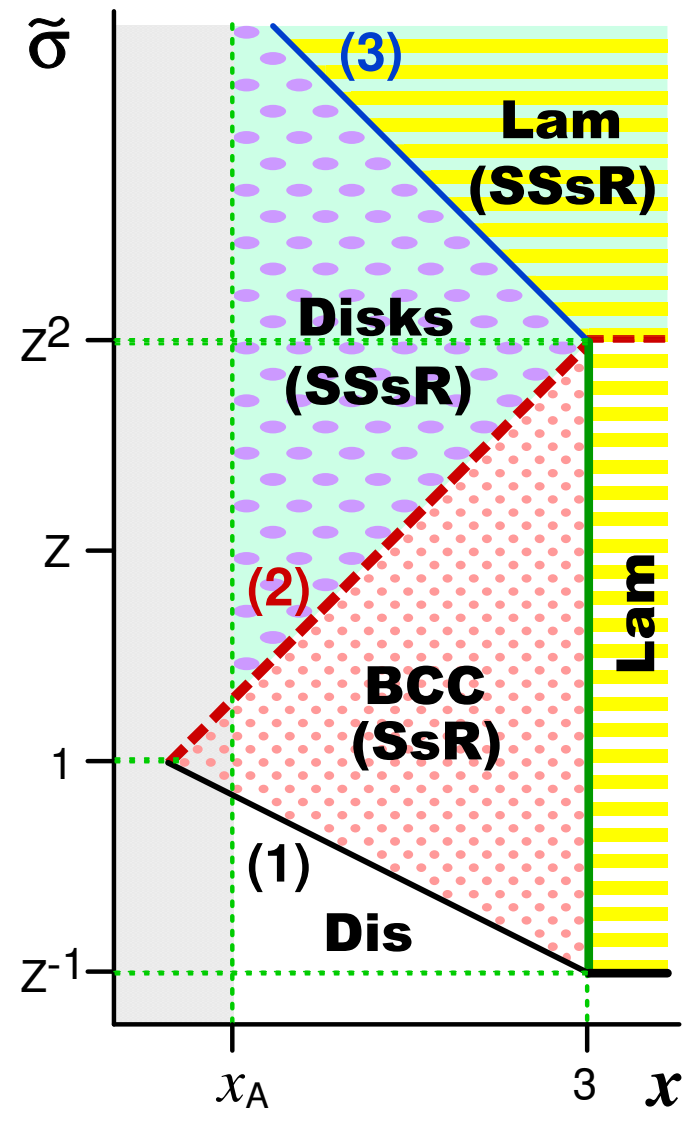

Fig.3

(a)

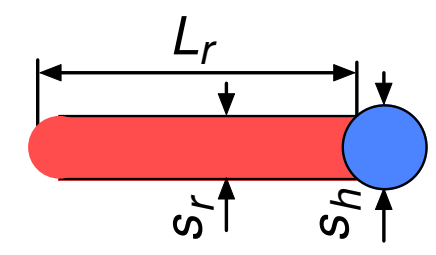

(b)

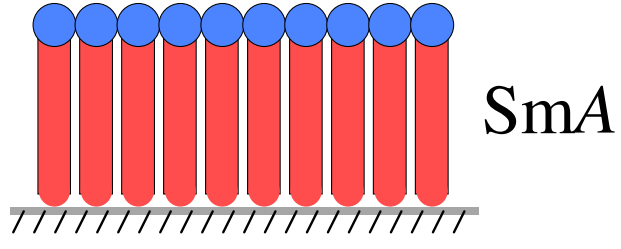
surface layer

(c)
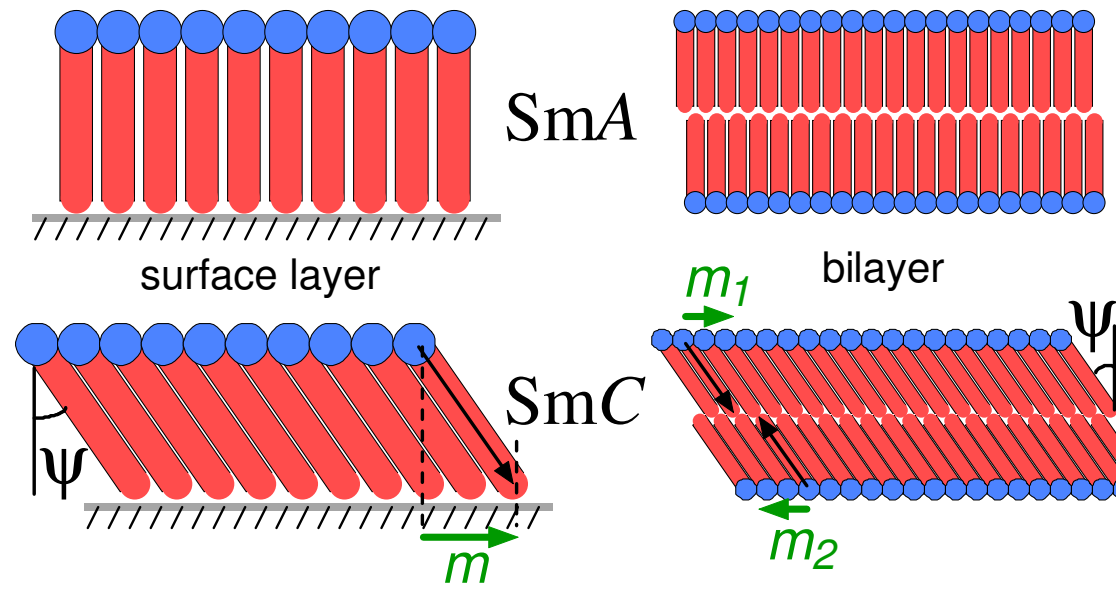
$m_{1} \quad$ bilayer

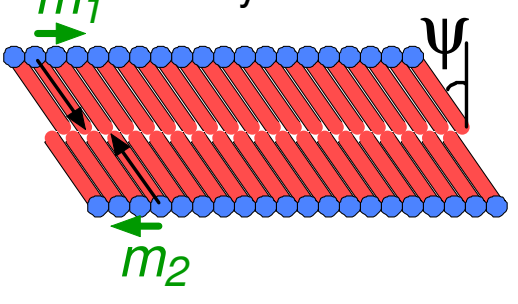

Fig.4 


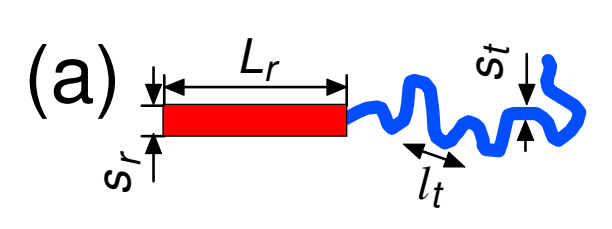

(b) $N$ ?

(C)
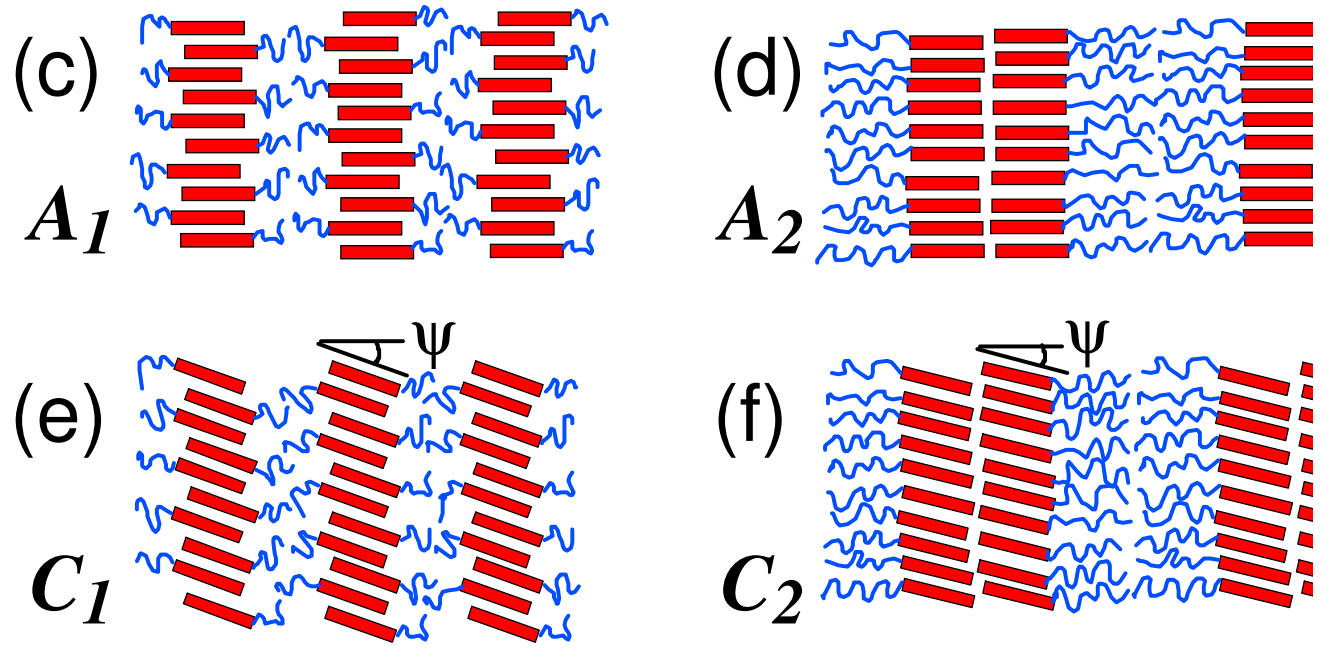

Fig.5

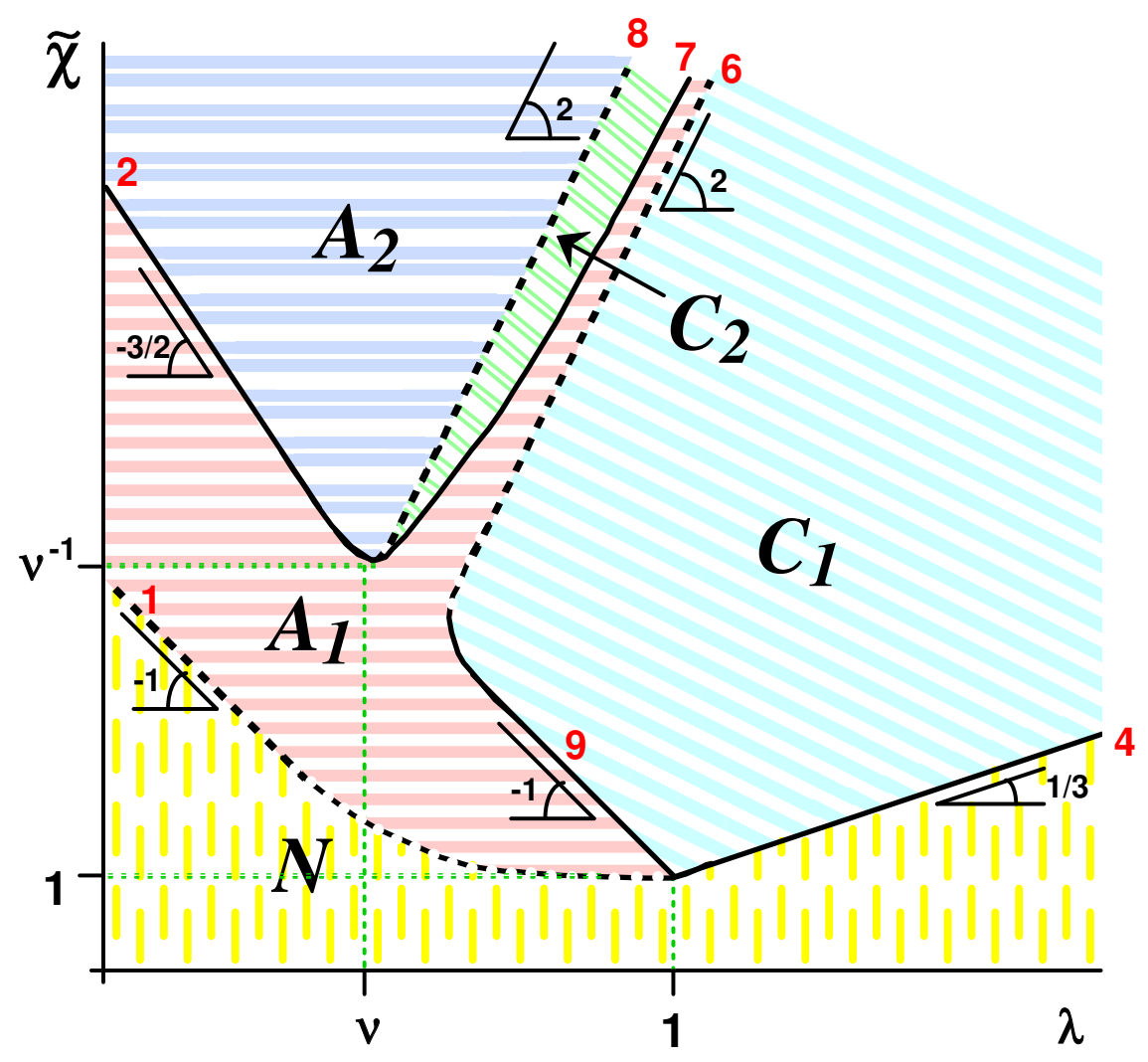

Fig.6 
(a)

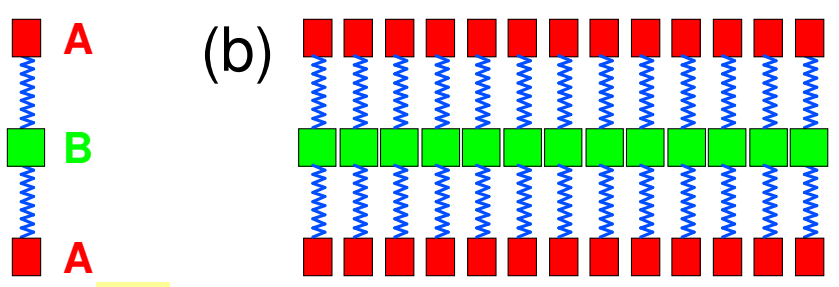

(c)

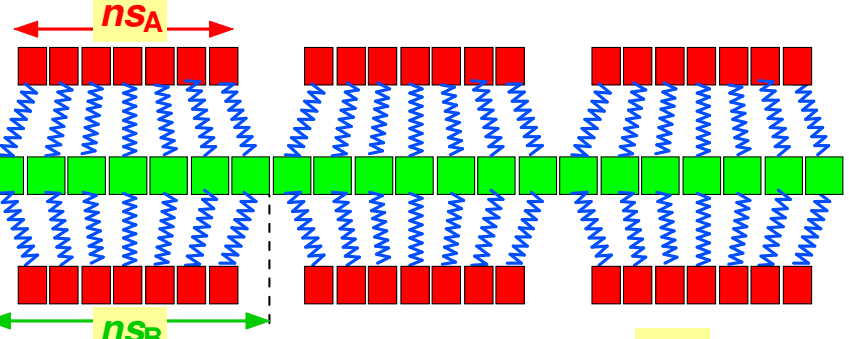

(d)

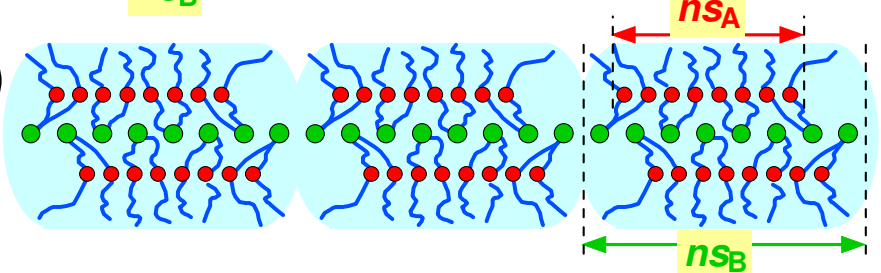

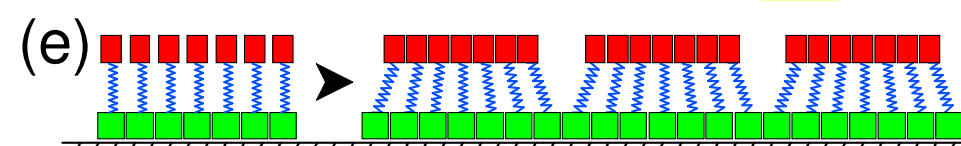

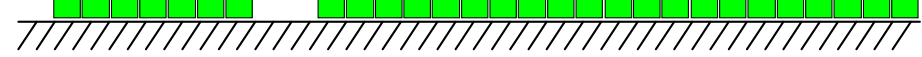

Fig.7

(a)

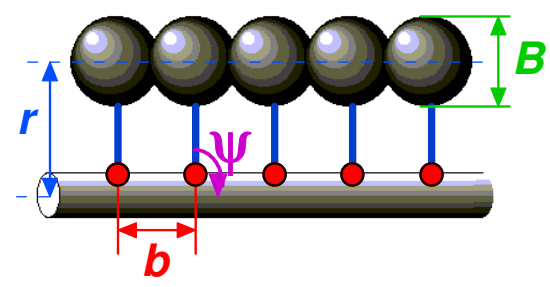

(c) 91TPT

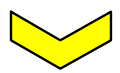

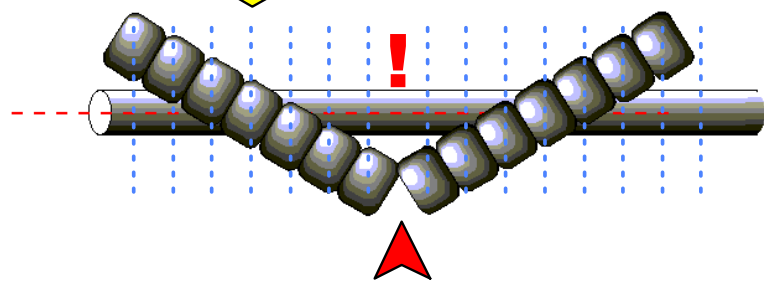

(b)
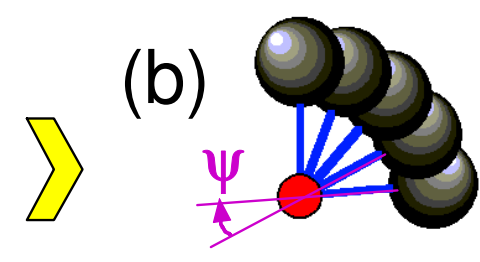

(d)

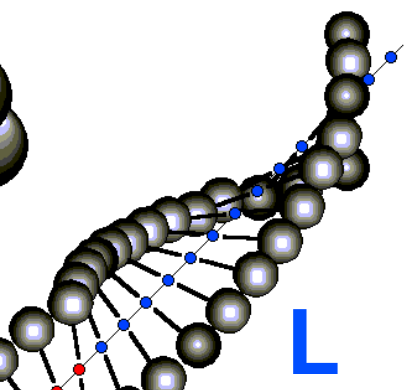

Fig.8 


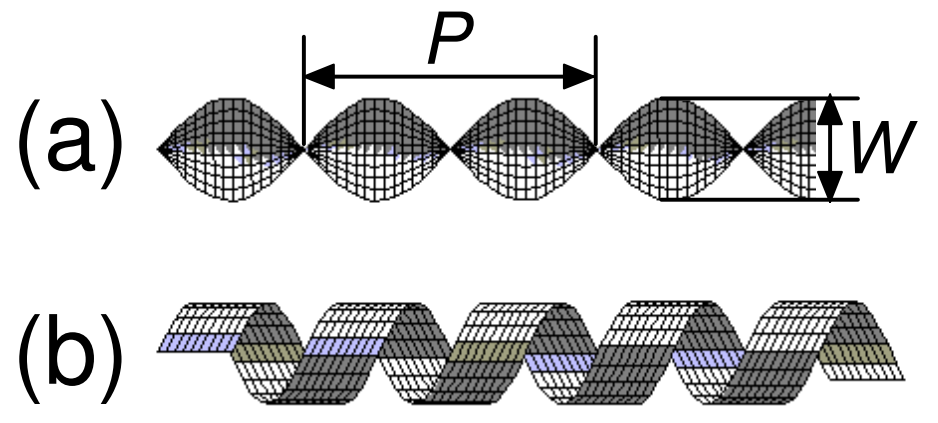

Fig.9

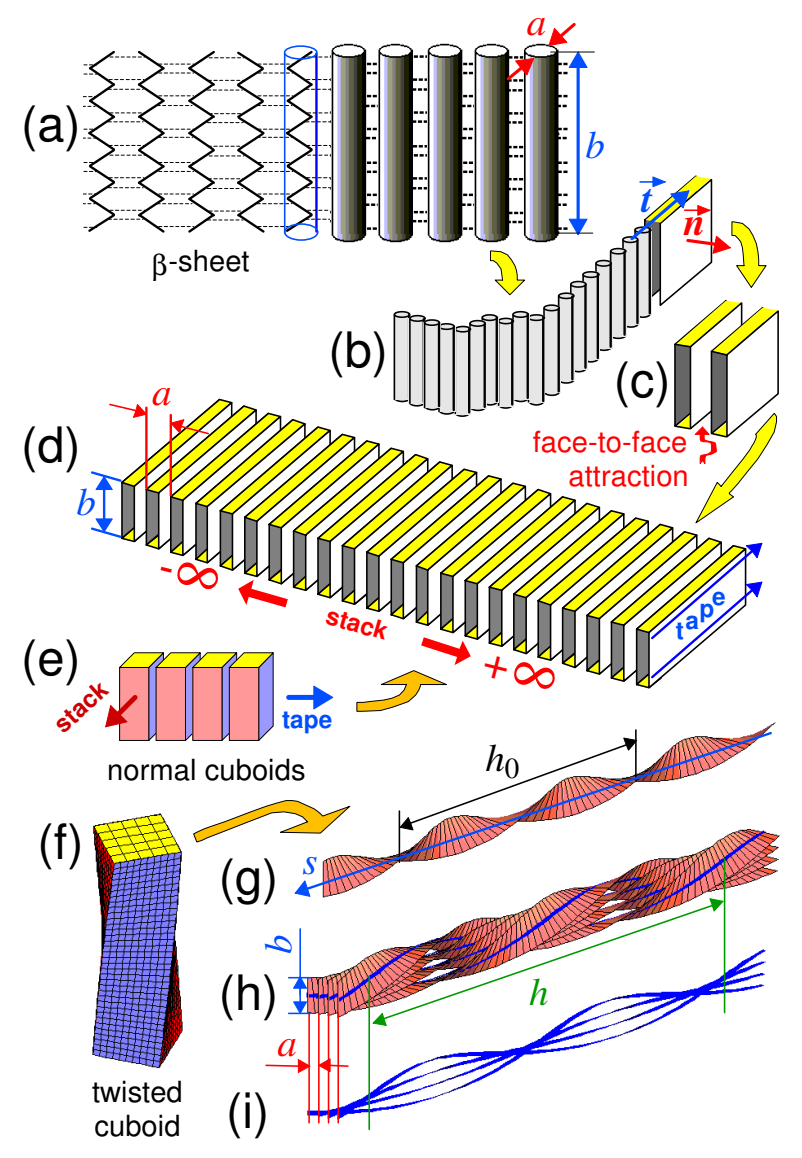

Fig.10 


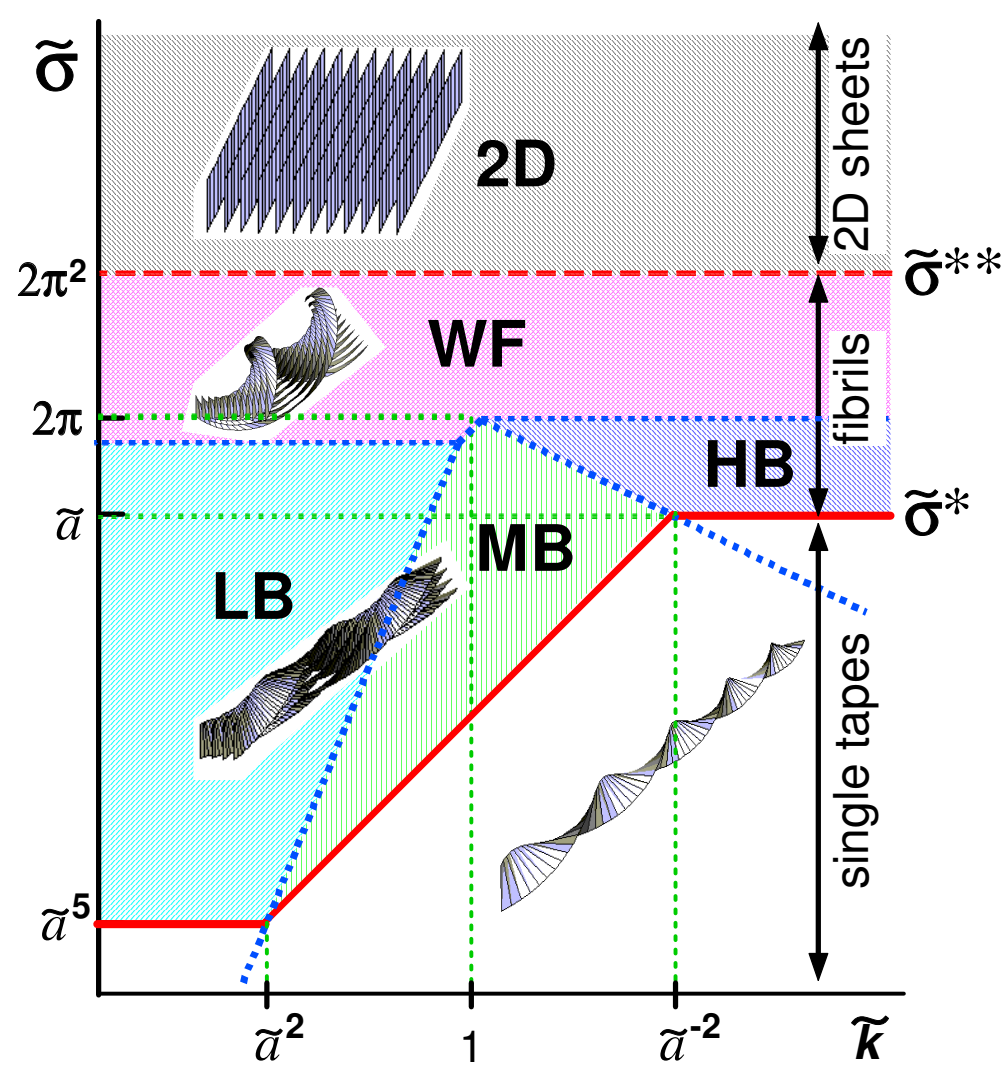

Fig.11

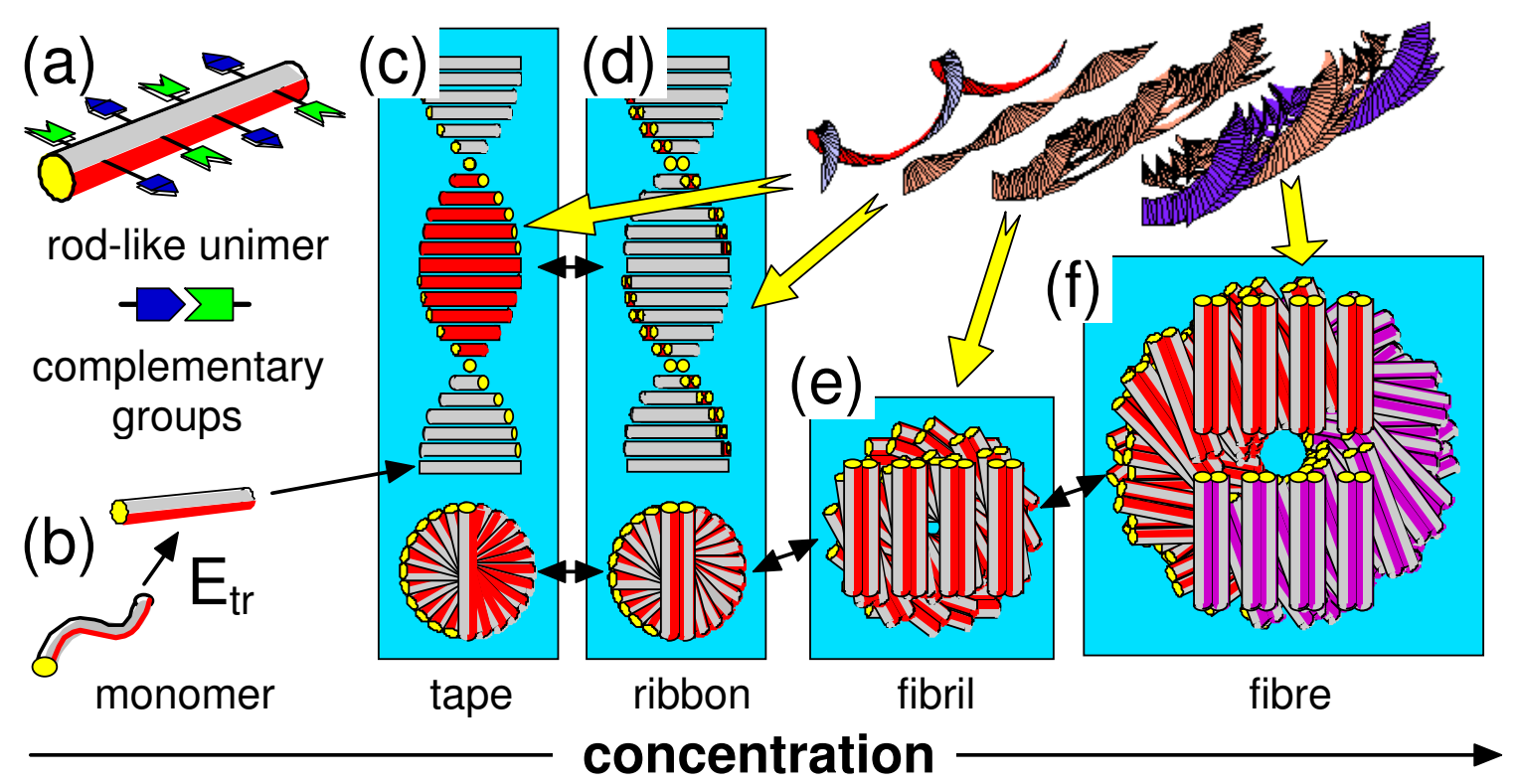

Fig. 12 

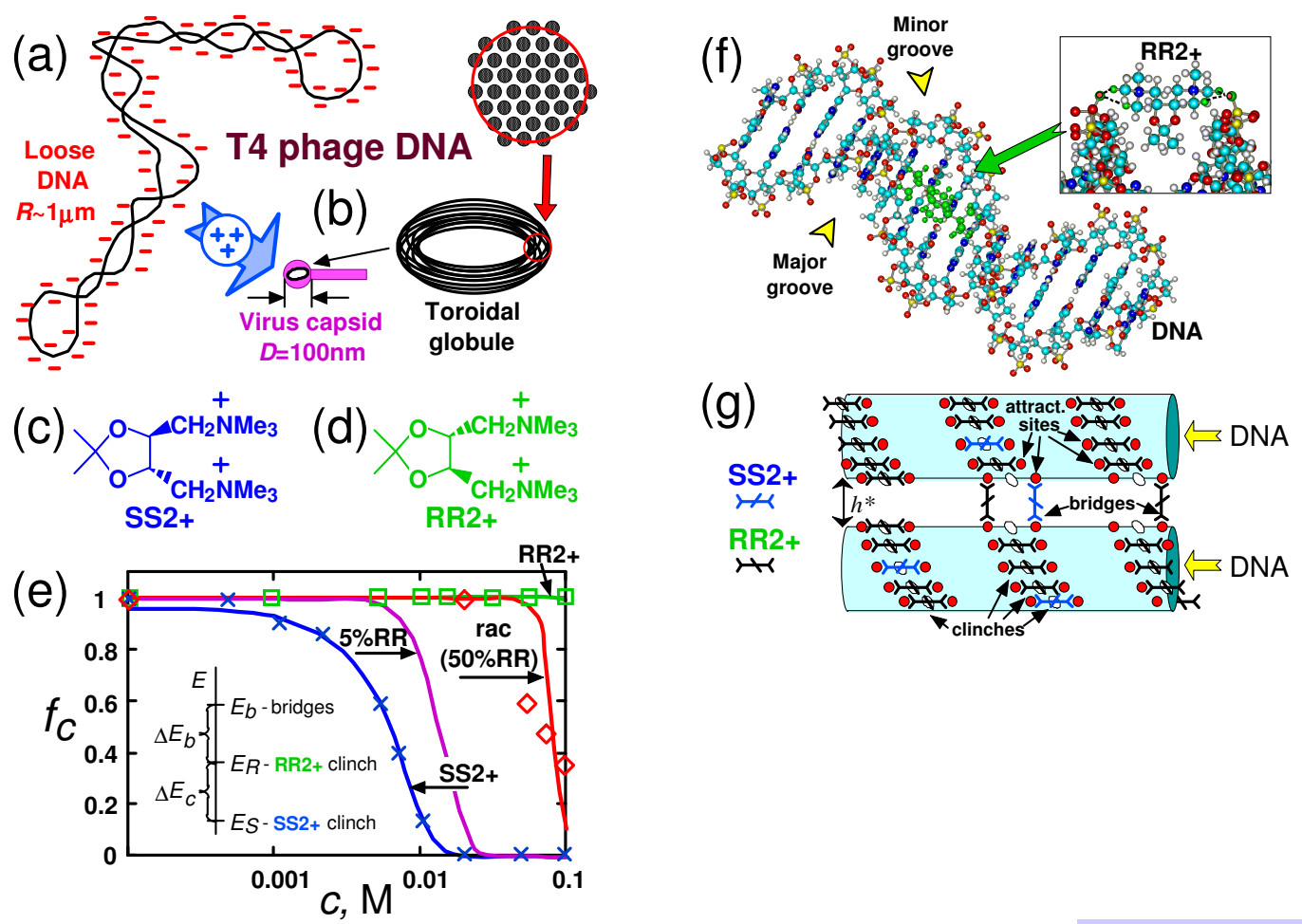

Fig.13
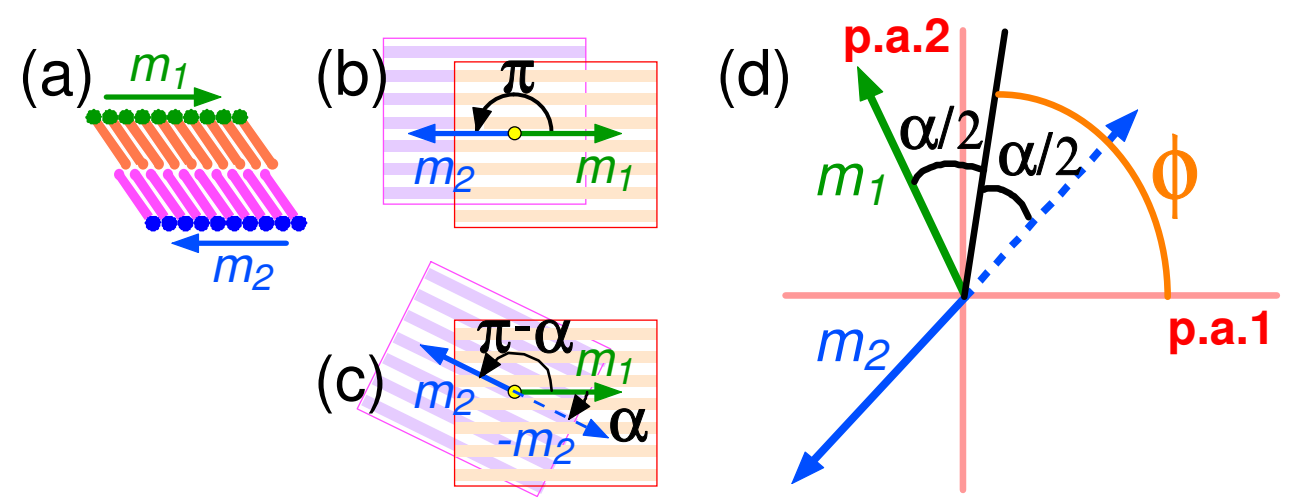

Fig.14 

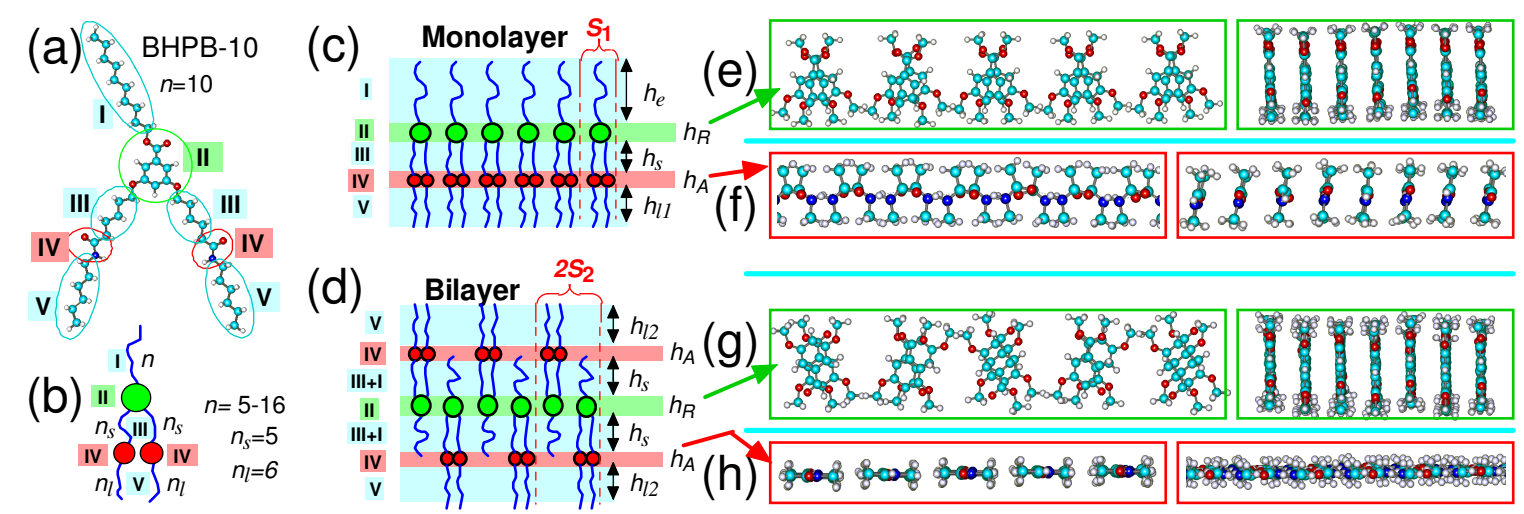

(d)

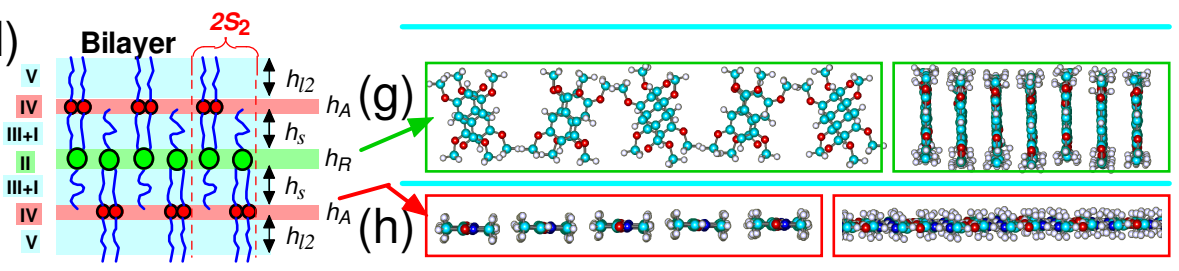

Fig.15

(a)
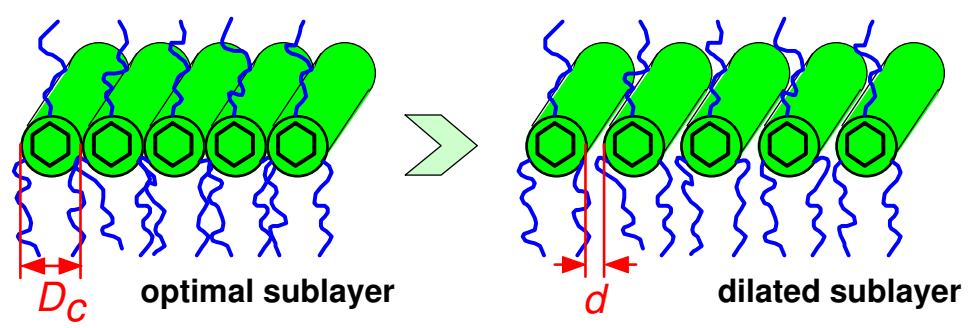

(b) infinite layer ... m.

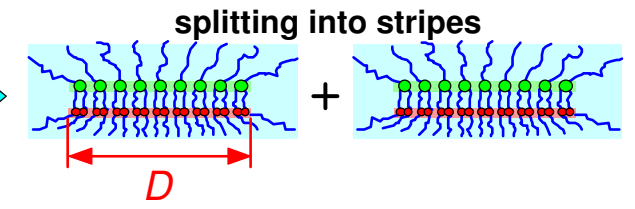

Fig.16 


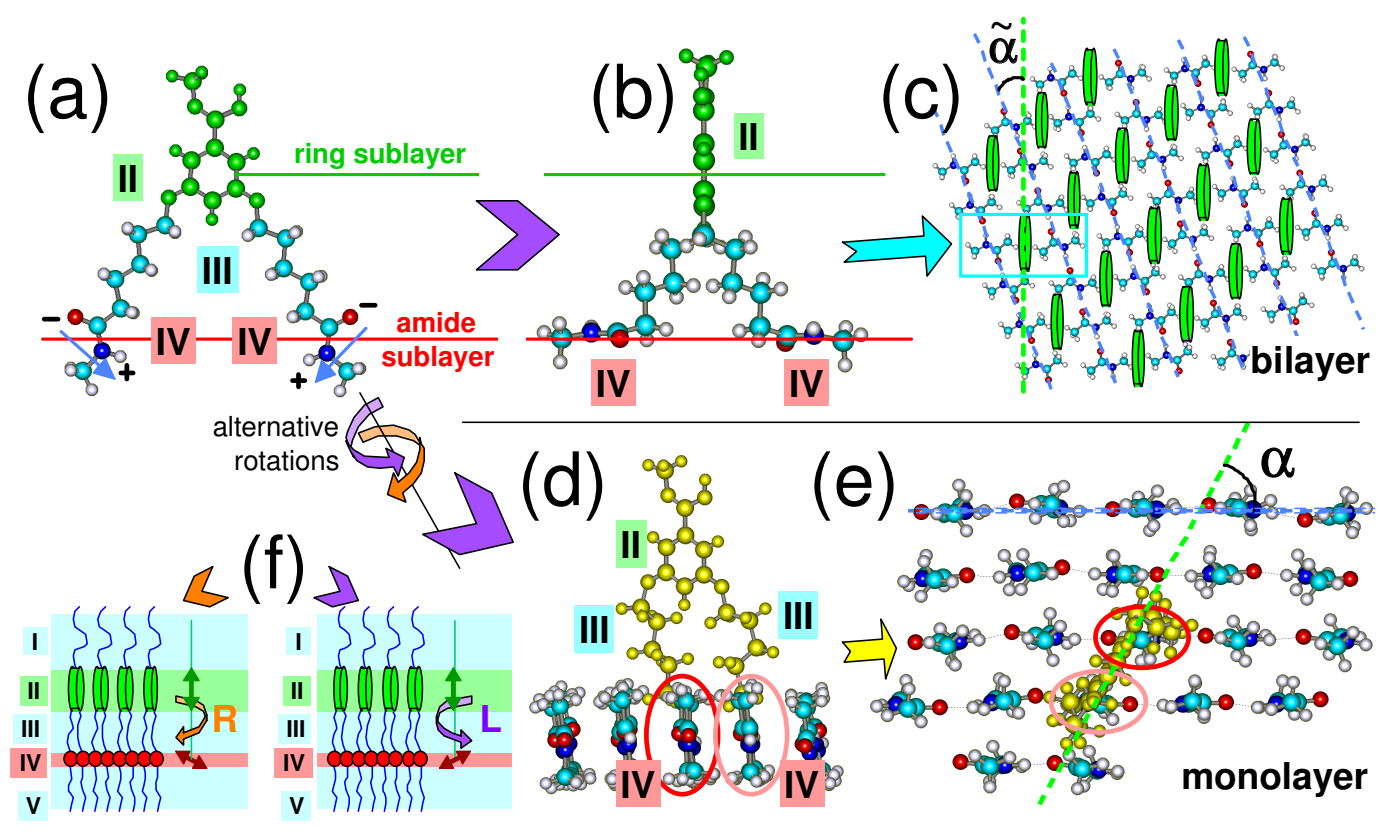

Fig. 17
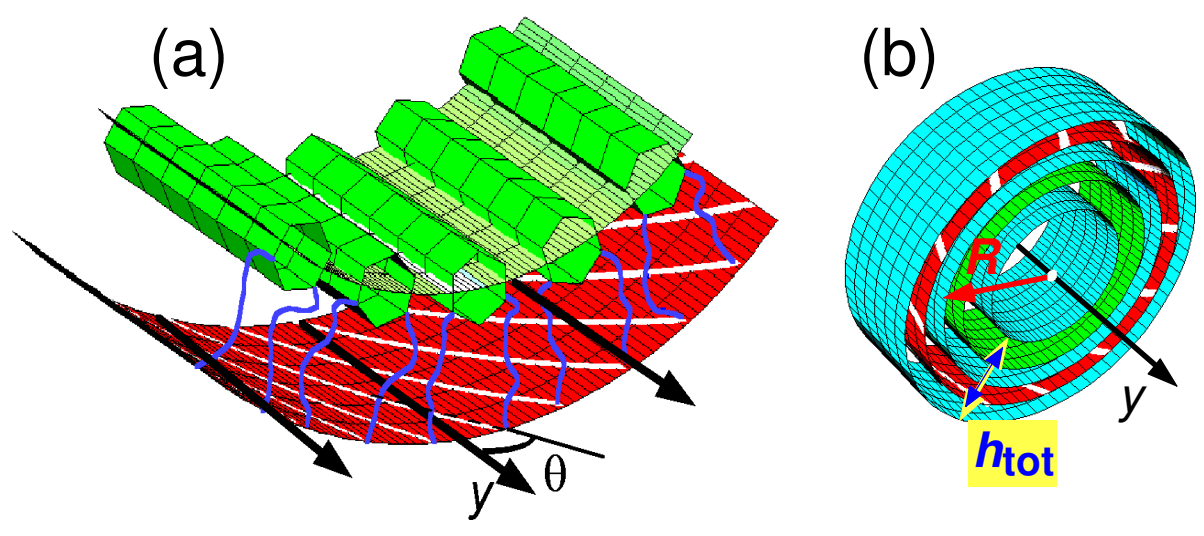

Fig. 18 
(a)

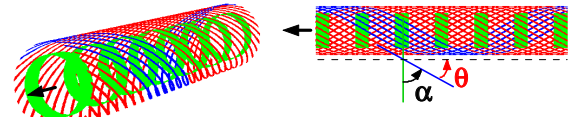

(b)

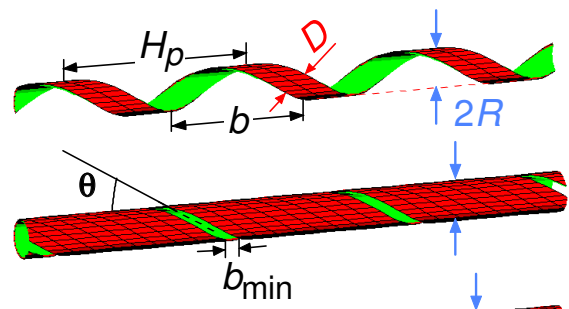

(d)

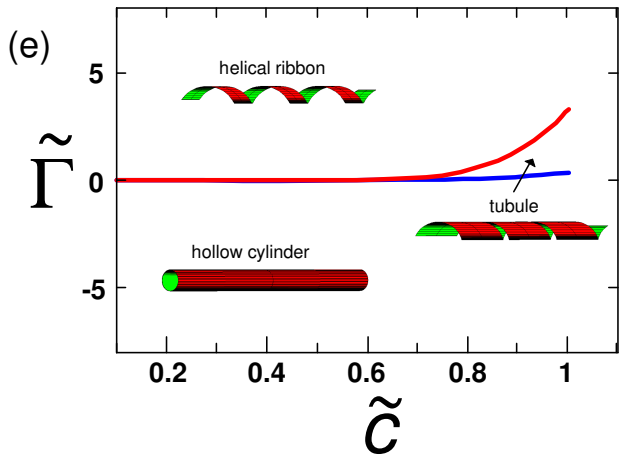

Fig.19

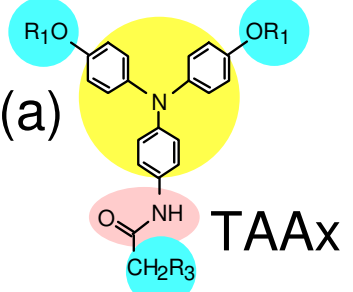

(b)
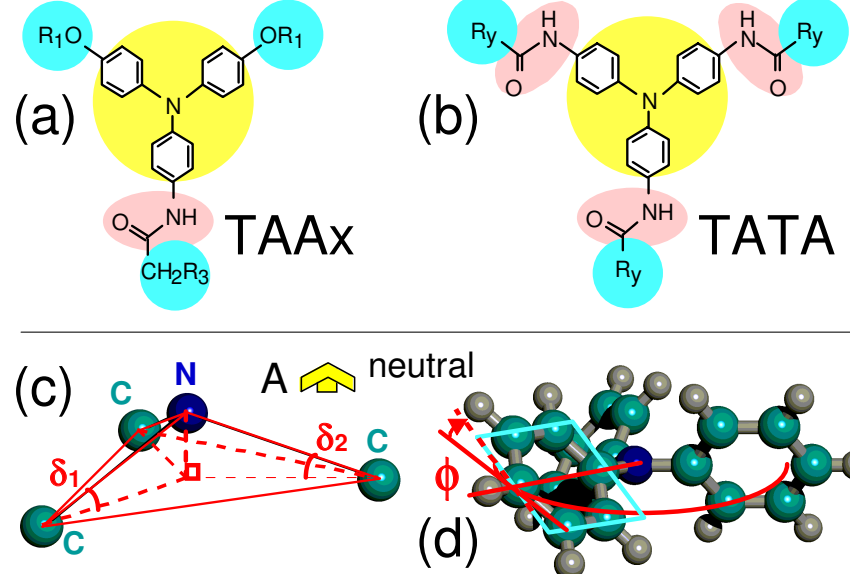

(d)

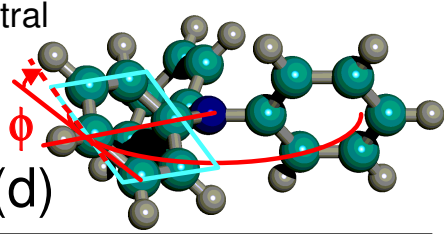

(e)

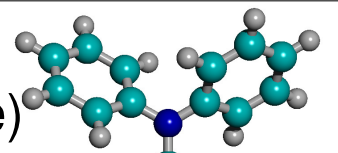

(f)

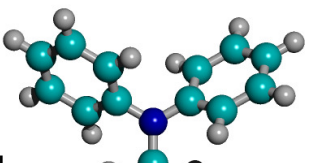

$D$ - 


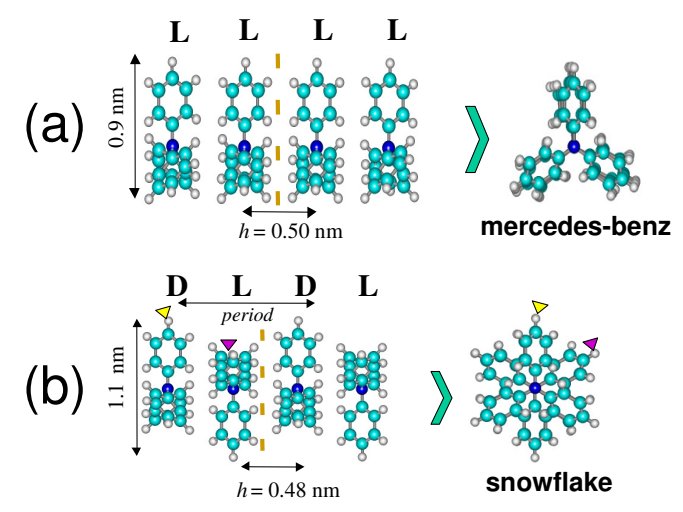

Fig. 21

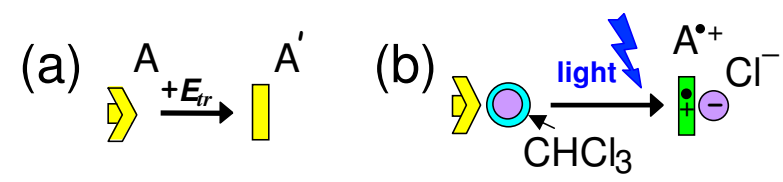

(C) tight column

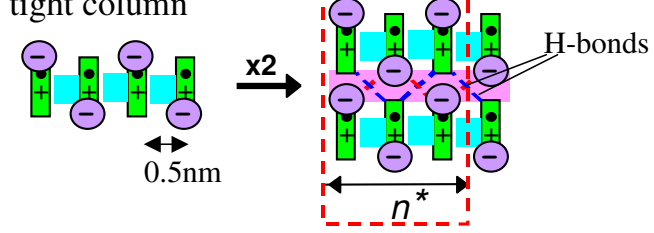




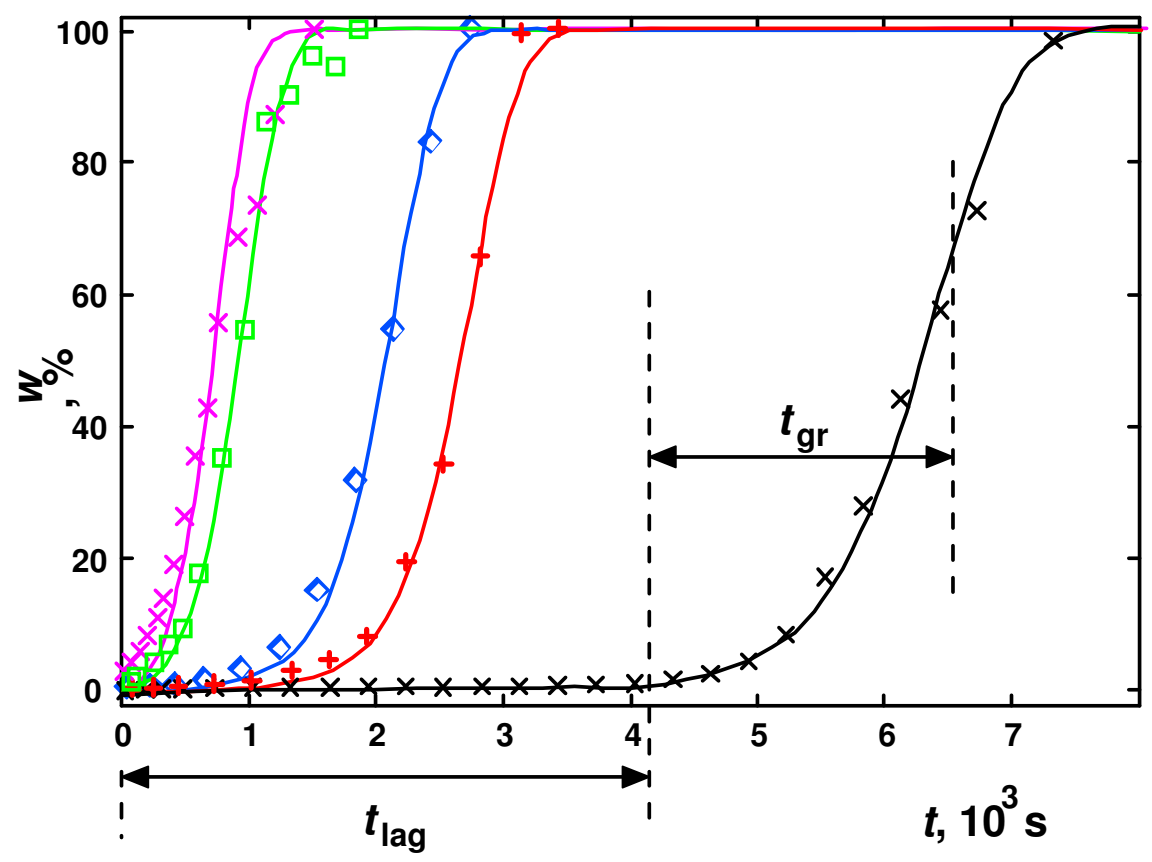

Fig.23

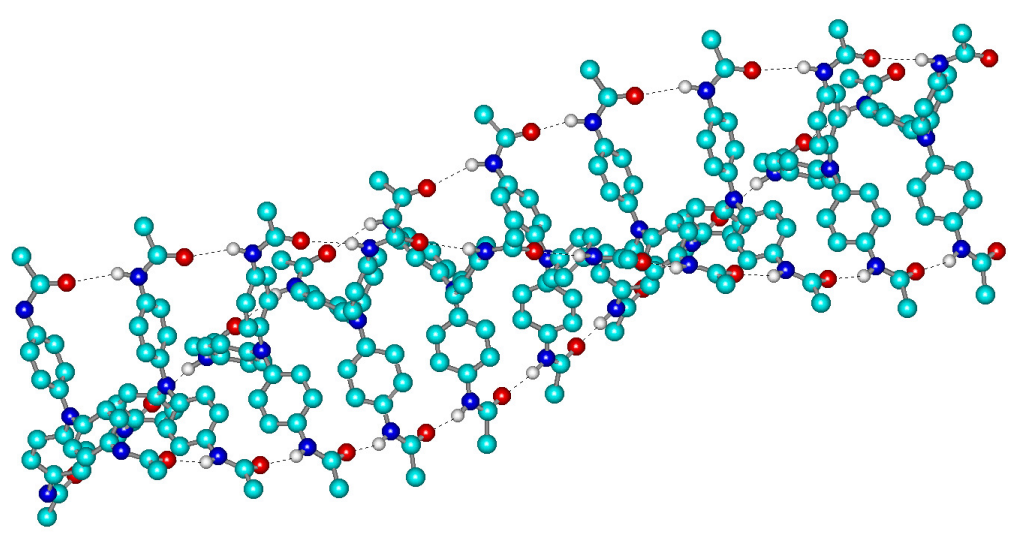

Fig.24 